[12] R. Ahlswede, "The capacity region of a channel with two senders and two receivers," Ann. Prob., pp. 805-814, Oct. 1974.

[13] A. B. Carliel, "A case where interference does not reduce capacity," IEEE Trans. Inf. Theory, vol. 21, pp. 569-570, Sep. 1975.

\section{Derivatives of Entropy Rate in Special Families of Hidden Markov Chains}

\author{
Guangyue Han and Brian Marcus, Fellow, IEEE
}

\begin{abstract}
Consider a hidden Markov chain obtained as the observation process of an ordinary Markov chain corrupted by noise. Recently Zuk $e t$ $a l$. showed how, in principle, one can explicitly compute the derivatives of the entropy rate of at extreme values of the noise. Namely, they showed that the derivatives of standard upper approximations to the entropy rate actually stabilize at an explicit finite time. We generalize this result to a natural class of hidden Markov chains called "Black Holes." We also discuss in depth special cases of binary Markov chains observed in binary-symmetric noise, and give an abstract formula for the first derivative in terms of a measure on the simplex due to Blackwell.
\end{abstract}

Index Terms-Analyticity, entropy, entropy rate, hidden Markov chain, hidden Markov model, hidden Markov process.

\section{INTRODUCTION}

Let $Y=\left\{Y_{-\infty}^{\infty}\right\}$ be a stationary Markov chain with a finite state alphabet $\{1,2, \ldots, B\}$. A function $Z=\left\{Z_{-\infty}^{\infty}\right\}$ of the Markov chain $Y$ with the form $Z=\Phi(Y)$ is called a hidden Markov chain; here $\Phi$ is a finite-valued function defined on $\{1,2, \ldots, B\}$, taking values in $\{1,2, \ldots, A\}$. Let $\Delta$ denote the probability transition matrix for $Y$; it is well known that the entropy rate $H(Y)$ of $Y$ can be analytically expressed using the stationary vector of $Y$ and $\Delta$. Let $W$ be the simplex, comprising the vectors

$$
\left\{w=\left(w_{1}, w_{2}, \ldots, w_{B}\right) \in \mathbb{R}^{B}: w_{i} \geq 0, \sum_{i} w_{i}=1\right\}
$$

and let $W_{a}$ be all $w \in W$ with $w_{i}=0$ for $\Phi(i) \neq a$. For $a \in A$, let $\Delta_{a}$ denote the $B \times B$ matrix such that $\Delta_{a}(i, j)=\Delta(i, j)$ for $j$ with $\Phi(j)=a$, and $\Delta_{a}(i, j)=0$ otherwise. For $a \in A$, define the scalar-valued and vector-valued functions $r_{a}$ and $f_{a}$ on $W$ by

$$
r_{a}(w)=w \Delta_{a} \mathbf{1}
$$

and

$$
f_{a}(w)=w \Delta_{a} / r_{a}(w)
$$

Note that $f_{a}$ defines the action of the matrix $\Delta_{a}$ on the simplex $W$.

If $Y$ is irreducible, it turns out that the entropy rate

$$
H(Z)=-\int \sum_{a} r_{a}(w) \log r_{a}(w) d Q(w)
$$

Manuscript received April 14, 2006; revised January 20, 2007. The material in this correspondence was presented at the IEEE International Symposium on Information Theory, Seattle, WA, July 2006.

The authors are with the Department of Mathematics, University of British Columbia, Vancouver, BC V6T 1Z2, Canada (e-mail: ghan@math.ubc.ca; marcus@math.ubc.ca).

Communicated by Y. Steinberg, Associate Editor for Shannon Theory.

Digital Object Identifier 10.1109/TIT.2007.899467 where $Q$ is Blackwell's measure [1] on $W$. This measure is defined as the limiting distribution $p\left(y_{0}=\cdot \mid z_{-\infty}^{0}\right)$.

Recently, there has been a great deal of work on the entropy rate of a hidden Markov chain. Jacquet et al. [6] considered entropy rate of the hidden Markov chain $Z$, obtained by passing a binary Markov chain through a binary-symmetric channel with crossover probability $\varepsilon$, and computed the derivative of $H(Z)$ with respect to $\varepsilon$ at $\varepsilon=0$. For the same channel, Ordentlich and Weissman used Blackwell's measure to bound the entropy rate [11] and obtained an asymptotical formula for entropy rate [12]. For certain more general channels, Zuk et al. [16], [17] proved a "stabilizing" property of the derivatives of entropy rate of a hidden Markov chain and computed the Taylor series expansion for a special case. Several authors have observed that the entropy rate of a hidden Markov chain can be viewed as the top Lyapunov exponent of a random matrix product [5], [6], [3]. Under mild positivity assumptions, Han and Marcus [4] showed the entropy rate of a hidden Markov chain varies analytically as a function of the underlying Markov chain parameters.

In Section II, we establish a "stabilizing" property for the derivatives of the entropy rate in a family we call "Black Holes." Using this property, one can, in principle, explicitly calculate the derivatives of the entropy rate for this case, generalizing the results of [16], [17].

In Section III, we consider binary Markov chains corrupted by binary-symmetric noise. For this class, we obtain results on the support of Blackwell's measure, and for a special case, that we call the "nonoverlapping" case, we express the first derivative of the entropy rate as the sum of terms, involving Blackwell's measure, which have meaningful interpretations.

\section{Stabilizing Property of Derivatives in Black Hole Case}

Suppose that for every $a \in A, \Delta_{a}$ is a rank one matrix, and every column of $\Delta_{a}$ is either strictly positive or all zeros. In this case, the image of $f_{a}$ is a single point and each $f_{a}$ is defined on the whole simplex $W$. Thus, we call this the Black Hole case. Analyticity of the entropy rate at a Black Hole follows from Theorem 1.1 of [4].

As an example, consider a binary-symmetric channel with crossover probability $\varepsilon$. Let $\left\{X_{n}\right\}$ be the input Markov chain with the transition matrix

$$
\Pi=\left[\begin{array}{ll}
\pi_{00} & \pi_{01} \\
\pi_{10} & \pi_{11}
\end{array}\right] .
$$

At time $n$ the channel can be characterized by the following equation:

$$
Z_{n}=X_{n} \oplus E_{n}
$$

where $\oplus$ denotes binary addition, $E_{n}$ denotes the independent and identically distributed (i.i.d.) binary noise with $p_{E}(0)=1-\varepsilon$ and $p_{E}(1)=\varepsilon$, and $Z_{n}$ denotes the corrupted output. Then $Y_{n}=\left(X_{n}, E_{n}\right)$ is jointly Markov, so $\left\{Z_{n}=\Phi\left(Y_{n}\right)\right\}$ is a hidden Markov chain with the corresponding

$$
\Delta=\left[\begin{array}{llll}
\pi_{00}(1-\varepsilon) & \pi_{00} \varepsilon & \pi_{01}(1-\varepsilon) & \pi_{01} \varepsilon \\
\pi_{00}(1-\varepsilon) & \pi_{00} \varepsilon & \pi_{01}(1-\varepsilon) & \pi_{01} \varepsilon \\
\pi_{10}(1-\varepsilon) & \pi_{10} \varepsilon & \pi_{11}(1-\varepsilon) & \pi_{11} \varepsilon \\
\pi_{10}(1-\varepsilon) & \pi_{10} \varepsilon & \pi_{11}(1-\varepsilon) & \pi_{11} \varepsilon
\end{array}\right] ;
$$

here, $\Phi$ maps states 1 and 4 to 0 and maps states 2 and 3 to 1 (the reader should not confuse $\Pi$ with the $4 \times 4$ matrix $\Delta$, which defines the hidden Markov chain via a deterministic function). When $\varepsilon=0$

$$
\Delta=\left[\begin{array}{llll}
\pi_{00} & 0 & \pi_{01} & 0 \\
\pi_{00} & 0 & \pi_{01} & 0 \\
\pi_{10} & 0 & \pi_{11} & 0 \\
\pi_{10} & 0 & \pi_{11} & 0
\end{array}\right]
$$


In this case, the nonzero entries of $\Delta_{0}$ and $\Delta_{1}$ are restricted to a single column and so both $\Delta_{0}$ and $\Delta_{1}$ have rank one. If $\pi_{i j}$ 's are all positive, then this is a Black Hole case.

For this channel, Zuk, et al. [17] and Ordentlich and Weissman [13] proved the "stabilizing" property of the derivatives of $H(Z)$ with respect to $\varepsilon$ at $\varepsilon=0$. We remark that some other special kinds of channels, which are Black Holes, are treated too in [17] and Taylor series expansion of $H(Z)$ around $\varepsilon=0$ were computed in [16]. In this section, we show that, in general, the coefficients of a Taylor series expansion, centered at a Black Hole, can be explicitly computed.

Suppose that $\Delta$ is analytically parameterized by a vector variable $\varepsilon=\left(\varepsilon_{1}, \varepsilon_{2}, \ldots, \varepsilon_{m}\right)$. For any smooth function $f$ of $\varepsilon$ and $\vec{n}=\left(n_{1}, n_{2}, \ldots, n_{m}\right) \in \mathbb{Z}_{+}^{m}$, define

$$
f^{(\vec{n})}=\frac{\partial^{|\vec{n}|} f}{\partial \varepsilon_{1}^{n_{1}} \partial \varepsilon_{2}^{n_{2}} \cdots \partial \varepsilon_{m}^{n_{m}}} ;
$$

here $|\vec{n}|$ denotes the order of the $\vec{n}$ th derivative of $f$ with respect to $\varepsilon$, and is defined as

$$
|\vec{n}|=n_{1}+n_{2}+\cdots+n_{m} .
$$

For $\vec{n}=\vec{l}_{1}+\vec{l}_{2}+\cdots+\vec{l}_{k}$, where $\vec{l}_{j}=\left(l_{j, 1}, l_{j, 2}, \ldots, l_{j, m}\right) \in \mathbb{Z}_{+}^{m}$, define

$$
\frac{\vec{n} !}{\overrightarrow{l_{1}} ! \vec{l}_{2} ! \cdots \vec{l}_{k} !}=\prod_{i} \frac{n_{i} !}{l_{1, i} ! l_{2, i} ! \cdots l_{k, i} !} .
$$

We say $\vec{l} \preceq \vec{n}$, if every component of $\vec{l}$ is less or equal to the corresponding one of $\vec{n}$, and $\vec{l} \prec \vec{n}$ if $\vec{l} \preceq \vec{n}$ and at least one component of $\vec{l}$ is strictly less than the corresponding one of $\vec{n}$. For $\vec{l} \preceq \vec{n}$, define

$$
C_{\vec{n}}^{\vec{l}}=\frac{\vec{n} !}{\vec{l} !(\vec{n}-\vec{l}) !}
$$

Let $H_{n}(Z)=H\left(Z_{0} \mid Z_{-n}^{-1}\right)$. It is well known that $H(Z)=$ $\lim _{n \rightarrow \infty} H_{n}(Z)$. The following theorem says that at a Black Hole, one can calculate the derivatives of $H(Z)$ with respect to $\varepsilon$ by taking the derivatives of $H_{n}(Z)$ with respect to $\varepsilon$ for large enough $n$.

Theorem 2.1: If at $\varepsilon=\hat{\varepsilon}$, for every $a \in A, \Delta_{a}$ is a rank-one matrix, and every column of $\Delta_{a}$ is either a positive or a zero column, then for $\vec{n}=\left(n_{1}, n_{2}, \ldots, n_{m}\right)$

$$
\left.H(Z)^{(\vec{n})}\right|_{\varepsilon=\hat{\varepsilon}}=\left.H_{|\vec{n}|}(Z)^{(\vec{n})}\right|_{\varepsilon=\hat{\varepsilon}} .
$$

In fact, we give a stronger result, Theorem 2.5 , later in this section.

Proof: For simplicity we drop $\varepsilon$ when the implication is clear from the context.

We shall first prove that for all sequences $z_{-\infty}^{0}$, the $\vec{n}$ th derivative of $p\left(z_{0} \mid z_{-\infty}^{-1}\right)$ stabilizes

$$
p^{(\vec{n})}\left(z_{0} \mid z_{-\infty}^{-1}\right)=p^{(\vec{n})}\left(z_{0} \mid z_{-|\vec{n}|-1}^{-1}\right), \quad \text { at } \varepsilon=\hat{\varepsilon} .
$$

Since $p\left(z_{0} \mid z_{-\infty}^{-1}\right)=p\left(y_{-1}=\cdot \mid z_{-\infty}^{-1}\right) \Delta_{z_{0}} \mathbf{1}$ (here $\cdot$ represent the states of the Markov chain $Y$, thus $p\left(y_{-1}=\cdot \mid z_{-\infty}^{-1}\right)$ is a row vector), it suffices to prove that for the $\vec{n}$ th derivative of $x_{i}=p\left(y_{i}=\cdot \mid z_{-\infty}^{i}\right)$, we have

$$
x_{i}^{(\vec{n})}=p^{(\vec{n})}\left(y_{i}=\cdot \mid z_{-\infty}^{i}\right)=p^{(\vec{n})}\left(y_{i}=\cdot \mid z_{i-|\vec{n}|}^{i}\right), \quad \text { at } \varepsilon=\hat{\varepsilon} .
$$

Consider the iteration

$$
x_{i}=\frac{x_{i-1} \Delta_{z_{i}}}{x_{i-1} \Delta_{z_{i}} \mathbf{1}} .
$$

In other words, $x_{i}$ can be viewed as a function of $x_{i-1}$ and $\Delta_{z_{i}}$. Let $g$ denote this function. Since at $\varepsilon=\hat{\varepsilon}, \Delta_{z_{i}}$ is a rank-one matrix, we conclude that $g$ is constant as a function of $x_{i-1}$. Thus, at $\varepsilon=\hat{\varepsilon}$

$$
\begin{aligned}
x_{i} & =p\left(y_{i}=\cdot \mid z_{-\infty}^{i}\right) \\
& =\frac{x_{i-1} \Delta_{z_{i}}}{x_{i-1} \Delta_{z_{i}} \mathbf{1}}=\frac{p\left(y_{i-1}=\cdot\right) \Delta_{z_{i}}}{p\left(y_{i-1}=\cdot\right) \Delta_{z_{i}} \mathbf{1}}=p\left(y_{i}=\cdot \mid z_{i}\right)
\end{aligned}
$$

where the third equality follows from the fact that $g$ is a constant as a function of $x_{i-1}$.

When $|\vec{n}|=1$, we have at $\varepsilon=\hat{\varepsilon}$

$x_{i}^{(\vec{n})}=\left.\frac{\partial g}{\partial \Delta_{z_{i}}}\right|_{\varepsilon=\hat{\varepsilon}}\left(x_{i-1}, \Delta_{z_{i}}\right) \Delta_{z_{i}}^{(\vec{n})}+\left.\frac{\partial g}{\partial x_{i-1}}\right|_{\varepsilon=\hat{\varepsilon}}\left(x_{i-1}, \Delta_{z_{i}}\right) x_{i-1}^{(\vec{n})}$.

Since at $\varepsilon=\hat{\varepsilon}, g$ is a constant as a function of $x_{i-1}$, we have

$$
\left.\frac{\partial g}{\partial x_{i-1}}\right|_{\varepsilon=\hat{\varepsilon}}\left(x_{i-1}, \Delta_{z_{i}}\right)=\frac{\partial(\text { a constant vector })}{\partial x_{i-1}}=\mathbf{0} .
$$

It then follows from (2.5) that at $\varepsilon=\hat{\varepsilon}$

$$
x_{i}^{(\vec{n})}=p^{(\vec{n})}\left(y_{i}=\cdot \mid z_{-\infty}^{i}\right)=p^{(\vec{n})}\left(y_{i}=\cdot \mid z_{i-1}^{i}\right) .
$$

When $|\vec{n}|>1$, we have

$$
x_{i}^{(\vec{n})}=\left.\frac{\partial g}{\partial x_{i-1}}\right|_{\varepsilon=\hat{\varepsilon}}\left(x_{i-1}, \Delta_{z_{i}}\right) x_{i-1}^{(\vec{n})}+\text { other terms }
$$

where "other terms" involve only lower order (than $|\vec{n}|$ ) derivatives of $x_{i-1}$. By induction, we conclude that

$$
x_{i}^{(\vec{n})}=p^{(\vec{n})}\left(y_{i}=\cdot \mid z_{-\infty}^{i}\right)=p^{(\vec{n})}\left(y_{i}=\cdot \mid z_{i-|\vec{n}|}^{i}\right) .
$$

at $\varepsilon=\hat{\varepsilon}$. We then have (2.4) and therefore (2.3) as desired.

By the proof of Theorem 1.1 of [4], the complexified $H_{n}(Z)$ uniformly converges to the complexified $H(Z)$, and so we can switch the limit operation and the derivative operation.

Thus, when $|\vec{l}|=1$, at all $\varepsilon$

$$
\begin{aligned}
H^{(\vec{l})}(Z) & =\left(\lim _{k \rightarrow \infty} \sum_{z_{-k}^{0}}\left(p\left(z_{-k}^{0}\right) \log p\left(z_{0} \mid z_{-k}^{-1}\right)\right)^{(\vec{l})}\right. \\
& =-\lim _{k \rightarrow \infty} \sum_{z_{-k}^{0}}\left(p^{(\vec{l})}\left(z_{-k}^{0}\right) \log p\left(z_{0} \mid z_{-k}^{-1}\right)+p\left(z_{-k}^{0}\right) \frac{p^{(\vec{l})}\left(z_{0} \mid z_{-k}^{-1}\right)}{p\left(z_{0} \mid z_{-k}^{-1}\right)}\right) .
\end{aligned}
$$

Since

$$
\sum_{z_{0}} p\left(z_{-k}^{0}\right) \frac{p^{(\vec{l})}\left(z_{0} \mid z_{-k}^{-1}\right)}{p\left(z_{0} \mid z_{-k}^{-1}\right)}=\sum_{z_{0}} p\left(z_{-k}^{-1}\right) p^{(\vec{l})}\left(z_{0} \mid z_{-k}^{-1}\right)=0
$$

we have for all $\varepsilon$

$$
H^{(\vec{l})}(Z)=-\lim _{k \rightarrow \infty} \sum_{z_{-k}^{0}}\left(p^{(\vec{l})}\left(z_{-k}^{0}\right) \log p\left(z_{0} \mid z_{-k}^{-1}\right)\right) .
$$

At $\varepsilon=\hat{\varepsilon}$, we obtain

$$
\begin{aligned}
H^{(\vec{l})}(Z) & =-\lim _{k \rightarrow \infty} \sum_{z_{-k}^{0}}\left(p^{(\vec{l})}\left(z_{-k}^{0}\right) \log p\left(z_{0} \mid z_{-1}\right)\right) \\
& =-\sum_{z_{-1}^{0}}\left(p^{(\vec{l})}\left(z_{-1}^{0}\right) \log p\left(z_{0} \mid z_{-1}\right)\right)=H_{1}^{(\vec{l})}(Z)
\end{aligned}
$$

which establishes the theorem in the case when $|\vec{n}|=1$. 
When $|\vec{n}|>1$, we fix any $\vec{l} \preceq \vec{n}$ with $|\vec{l}|=1$. Using the fact that we can interchange the order of limit and derivative operations and using (2.6) and multivariate Leibnitz formula, we have for all $\varepsilon$

$$
\begin{aligned}
H^{(\vec{n})} & (Z) \\
& =\left(H^{(\vec{l})}\right)^{(\vec{n}-\vec{l})}(Z) \\
& =-\lim _{k \rightarrow \infty} \sum_{z_{-k}^{0}} \sum_{\vec{j} \preceq \vec{n}-\vec{l}} C_{\vec{n}-\vec{l}}^{\vec{j}} p^{(\vec{l}+\vec{\jmath})}\left(z_{-k}^{0}\right)\left(\log p\left(z_{0} \mid z_{-k}^{-1}\right)\right)^{(\vec{n}-\vec{l}-\vec{\jmath})} .
\end{aligned}
$$

Note that the term $\left(\log p\left(z_{0} \mid z_{-k}^{-1}\right)\right)^{(\vec{n}-\vec{l}-\vec{\jmath})}$ involves only the lower order (less than or equal to $|\vec{n}|-1$ ) derivatives of $p\left(z_{0} \mid z_{-k}^{-1}\right)$, which are already "stabilizing" in the sense of (2.3); so, we have at $\varepsilon=\hat{\varepsilon}$

$$
\begin{aligned}
& H^{(\vec{n})}(Z) \\
& =-\sum_{z_{-|\vec{n}|}^{0}} \sum_{\vec{j} \preceq \vec{n}-\vec{l}} C_{\vec{n}-\vec{l}}^{\vec{j}} p^{(\vec{l}+\vec{\jmath})}\left(z_{-|\vec{n}|}^{0}\right)\left(\log p\left(z_{0} \mid z_{-|\vec{n}|}^{-1}\right)\right)^{(\vec{n}-\vec{l}-\vec{\jmath})} \\
& =H_{|\vec{n}|}^{(\vec{n})}(Z) .
\end{aligned}
$$

We thus prove the theorem.

Remark 2.2: It follows from (2.5) that a hidden Markov chain at a Black Hole is, in fact, a Markov chain. Note that in the argument above the proof of the stabilizing property of the first derivative (as opposed to higher derivatives) requires only that the hidden Markov chain is Markov and that we can interchange the order of limit and derivative operations (instead of the stronger Black Hole property). Therefore, if a hidden Markov chain $Z$ defined by $\hat{\Delta}$ and $\Phi$ is in fact a Markov chain, and the complexified $H_{n}(Z)$ uniformly converges to $H(Z)$ on some neighborhood of $\hat{\Delta}$ (e.g., if the conditions of Theorem 1.1, 6.1, or 7.5 of [4] hold), then at $\hat{\Delta}$, we have

$$
H^{\prime}(Z)=H_{1}^{\prime}(Z)
$$

For instance, consider the following hidden Markov chain $Z$ defined by

$$
\hat{\Delta}=\left[\begin{array}{ccc}
1 / 4 & 1 / 4 & 1 / 2 \\
0 & 1 / 6 & 5 / 6 \\
7 / 8 & 1 / 8 & 0
\end{array}\right]
$$

with $\Phi(1)=0$ and $\Phi(2)=\Phi(3)=1 . Z$ is in fact a Markov chain (see [7, p. 134]), and one checks that $\hat{\Delta}$ satisfies the conditions in Theorem 7.5 in [4]. We conclude that for this example, (2.7) holds.
In the cases studied in [16], [17], [13], the authors obtained, using a finer analysis, a shorter "stabilizing length." This shorter length can be derived for the Black Hole case as well, as shown in Theorem 2.5 below, even though the proof in [17] does not seem to work.

We need some preliminary lemmas for the proof of Theorem 2.5.

We say $\vec{l}<\vec{n}$ if either $(|\vec{l}|<|\vec{n}|)$ or $(|\vec{l}|=|\vec{n}|$ and $\vec{l}$ is less than $\vec{n}$ lexicographically). By induction, one can prove that the formal derivative of $y \log y$ takes the following form of the first equation at the bottom of the page, where $E_{\left[\vec{a}_{1}, \vec{a}_{2}, \ldots, \vec{a}_{m+1}\right]}$ is a real number, denoting the corresponding coefficient. Let $q_{\vec{i}}[y]$ denote the "coefficient" of $y^{(\vec{i})}$, which is a function of $y$ and its formal derivatives (up to the $|\vec{i}|$ th order derivative). Thus, we have

$$
(y \log y)^{(\vec{N})}=\sum_{i=1}^{\vec{N}} q_{\vec{i}}[y] y^{(\vec{i})}=\operatorname{High}_{\vec{N}}[y]+\operatorname{Low}_{\vec{N}}[y]
$$

where

and

$$
\operatorname{High}_{\vec{N}}[y]=\sum_{\lceil(|\vec{N}|+1) / 2\rceil \leq|\vec{i}| \leq|\vec{N}|} q_{\vec{i}}[y] y^{(\vec{i})}
$$

$$
\operatorname{Low}_{\vec{N}}[y]=\sum_{|\vec{\imath}| \leq\lceil(|\vec{N}|-1) / 2\rceil} q_{\vec{i}}[y] y^{(\vec{i})} .
$$

For a sequence (without order)

$$
\mathbf{a}=\underbrace{a_{1}, \ldots, a_{1}}_{k_{1} \text { times }}, \underbrace{a_{2}, \ldots, a_{2}}_{k_{2} \text { times }}, \ldots, \underbrace{a_{l}, \ldots, a_{l}}_{k_{l} \text { times }},
$$

where $a_{j}$ 's are pairwise distinct, let $\langle\mathbf{a}\rangle=k_{1} ! k_{2} ! \cdots k_{l}$ !.

Lemma 2.3: When $\lceil(|\vec{N}|+1) / 2\rceil \leq|\vec{i}| \leq|\vec{N}|$

$$
q_{\vec{i}}[y]=C_{\vec{N}}^{\vec{i}}(\log y+1)^{(\vec{N}-\vec{\imath})} .
$$

Proof: In this proof, we use $f(y)$ to denote the function $y \log y$. Then by multivariate Faa Di Bruno formula [2], [8], we have the second equation at the bottom of the page, where we used the fact that

$$
\left\langle a_{1}, a_{2}, \ldots, a_{m}\right\rangle=\left\langle a_{2}, \ldots, a_{m}\right\rangle
$$

if $\left|\vec{a}_{1}\right| \geq\left\lceil\left(\left|\vec{a}_{1}+\cdots+\vec{a}_{m}\right|+1\right) / 2\right\rceil$. Bearing in mind that $f^{(1)}(y)=$ $\log y+1$, we prove the lemma.

$$
\begin{aligned}
(y \log y)^{(\vec{N})} & =\sum_{\vec{a}_{1} \geq \vec{a}_{2} \geq \cdots \geq \vec{a}_{m+1}: \vec{a}_{1}+\vec{a}_{2}+\cdots+\vec{a}_{m+1}=\vec{N}} E_{\left[\vec{a}_{1}, \vec{a}_{2}, \ldots, \vec{a}_{m+1}\right]} \frac{y^{\left(\vec{a}_{1}\right)} y^{\left(\vec{a}_{2}\right)} \cdots y^{\left(\vec{a}_{m+1}\right)}}{y^{m}}+y^{(\vec{N})}(\log y+1) \\
& =\sum_{\vec{i} \prec \vec{N}} y^{\left(\vec{a}_{1}=\vec{i}\right)} \sum_{\vec{a}_{2} \geq \vec{a}_{3} \geq \cdots \geq \vec{a}_{m+1}} E_{\left[\vec{a}_{1}, \vec{a}_{2}, \ldots, \vec{a}_{m+1}\right]} \frac{y^{\left(\vec{a}_{2}\right)} y^{\left(\vec{a}_{3}\right)} \cdots y^{\left(\vec{a}_{m+1}\right)}}{y^{m}}+y^{(\vec{N})}(\log y+1)
\end{aligned}
$$

$$
\begin{aligned}
f(y)^{(\vec{N})} & =\sum_{\vec{a}_{1} \geq \vec{a}_{2} \geq \cdots \geq \vec{a}_{m}: \vec{a}_{1}+\vec{a}_{2}+\cdots+\vec{a}_{m}=\vec{N}} f^{(m)}(y) \frac{1}{\left\langle\vec{a}_{1}, \vec{a}_{2}, \ldots, \vec{a}_{m}\right\rangle} \frac{\vec{N} !}{\vec{a}_{1} ! \vec{a}_{2} ! \cdots \vec{a}_{m} !} y^{\left(\vec{a}_{1}\right)} y^{\left(\vec{a}_{2}\right)} \cdots y^{\left(\vec{a}_{m}\right)} \\
& =\sum_{\Gamma(|\vec{N}|+1) / 2\rceil \leq\left|\vec{a}_{1}\right| \leq|\vec{N}|} C_{\vec{N}}^{\vec{a}_{1}} y^{\left(\vec{a}_{1}\right)} \sum_{\vec{a}_{2} \geq \cdots \geq \vec{a}_{m}: \vec{a}_{2}+\cdots+\vec{a}_{m}=\vec{N}-\vec{a}_{1}}\left(f^{(1)}\right)^{(m-1)}(y) \frac{1}{\left\langle\vec{a}_{2}, \ldots, \vec{a}_{m}\right\rangle} \frac{\left(\vec{N}-\vec{a}_{1}\right) !}{\vec{a}_{2} ! \cdots \vec{a}_{m} !} y^{\left(\vec{a}_{2}\right)} \cdots y^{\left(\vec{a}_{m}\right)}+\operatorname{Low}_{\vec{N}}[y] \\
& =\sum_{\Gamma(|\vec{N}|+1) / 2\rceil \leq\left|\vec{a}_{1}\right| \leq|\vec{N}|} C_{\vec{N}}^{\vec{a}_{1}} y^{\left(\vec{a}_{1}\right)}\left(f^{(1)}(y)\right)^{\vec{N}-\vec{a}_{1}}+\operatorname{Low}_{\vec{N}}[y]
\end{aligned}
$$




\section{Lemma 2.4:}

$$
\operatorname{Low}_{\vec{N}}[a x]=\sum_{|\vec{i}| \leq\lceil(|\vec{N}|-1) / 2\rceil} r_{\vec{i}}[a] x^{(\vec{i})}+\sum_{|\vec{i}| \leq\lceil(|\vec{N}|-1) / 2\rceil} s_{\vec{\imath}}[x] a^{(\vec{\imath})}
$$

where $r_{\vec{i}}[a]$ is a function of $a$ and its derivatives (up to order $\lceil(|\vec{N}|-$ $1) / 2\rceil$ ), and $s_{\vec{i}}[x]$ is a function of $x$ and its derivatives (up to order $\lceil(|\vec{N}|-1) / 2\rceil)$. Also

$$
s_{0}[x]=\operatorname{Low}_{\vec{N}}[x] .
$$

Proof: By multivariate Leibnitz formula, we have

$$
\begin{aligned}
& (a x \log (a x))^{(\vec{N})} \\
& =\sum_{\vec{i}+\vec{j} \preceq \vec{N}} \frac{\vec{i} !}{\vec{i} ! \vec{j} !(\vec{N}-\vec{i}-\vec{j}) !} a^{(\vec{i})} x^{(\vec{j})}(\log (a x))^{(\vec{N}-\vec{i}-\vec{j})}
\end{aligned}
$$

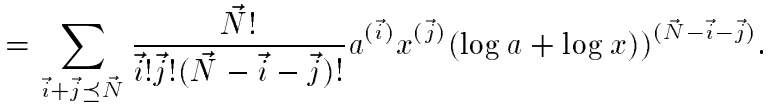

Thus, there exist a function of $a$ and its derivatives $t_{\vec{i}}[a]$, and a function of $x$ and its derivatives $w_{\vec{i}}[x]$ such that

$$
((a x) \log (a x))^{(\vec{N})}=\sum_{\vec{i} \preceq \vec{N}} t_{\vec{i}}[a] x^{(\vec{i})}+\sum_{\vec{i} \preceq \vec{N}} w_{\vec{i}}[x] a^{(\vec{i})}
$$

with $w_{0}[x]=(x \log x)^{(\vec{N})}$.

By Lemma 2.3, we have

$$
\begin{aligned}
& \operatorname{High}_{\vec{N}}[a x] \\
& \quad=\sum_{\lceil(|\vec{N}|+1) / 2\rceil \leq|\vec{i}| \leq|\vec{N}|} q_{\vec{i}}[a x](a x)^{(\vec{i})} \\
& =\sum_{\lceil(|\vec{N}|+1) / 2\rceil \leq|\vec{i}| \leq|\vec{N}|} C_{\vec{N}}^{\vec{n}}(\log a+\log x+1)^{(\vec{N}-\vec{i})}(a x)^{(\vec{i})} .
\end{aligned}
$$

Thus, we conclude that there exist a function of $a$ and its derivatives $u_{\vec{i}}[a]$, and a function of $x$ and its derivatives $v_{\vec{i}}[x]$ such that

$$
\operatorname{High}_{\vec{N}}[a x]=\sum_{\vec{i} \preceq \vec{N}} u_{i}[a] x^{(i)}+\sum_{\vec{i} \preceq \vec{N}} v_{\vec{i}}[x] a^{(\vec{i})}
$$

with $v_{0}[x]=\operatorname{High}_{\vec{N}}[x]$. Since

$$
\operatorname{Low}_{\vec{N}}[a x]=((a x) \log (a x))^{(\vec{N})}-\operatorname{High}_{\vec{N}}[a x]
$$

the existence of $r_{\vec{\imath}}[a]$ and $s_{\vec{\imath}}[x]$ then follows, and they depend on the derivatives only up to $\lceil(|\vec{N}|-1) / 2\rceil$, and $s_{0}[x]=$ Low $_{\vec{N}}[x]$.
Theorem 2.5: If at $\varepsilon=\hat{\varepsilon}$, for every $a \in A, \Delta_{a}$ is a rank-one matrix, and every column of $\Delta_{a}$ is either a positive or a zero column, then for $\vec{n}=\left(n_{1}, n_{2}, \ldots, n_{m}\right)$

$$
\left.H(Z)^{(\vec{n})}\right|_{\varepsilon=\hat{\varepsilon}}=\left.H_{\lceil(|\vec{n}|+1) / 2\rceil}(Z)^{(\vec{n})}\right|_{\varepsilon=\hat{\varepsilon}} .
$$

Proof: For simplicity, we drop $\varepsilon$ when the implication is clear from the context. Recall that

$$
\begin{aligned}
H_{n}(Z) & =-\sum_{z_{-n}^{0}} p\left(z_{-n}^{0}\right) \log p\left(z_{0} \mid z_{-n}^{-1}\right) \\
& =-\left(\sum_{z_{-n}^{0}} p\left(z_{-n}^{0}\right) \log p\left(z_{-n}^{0}\right)-\sum_{z_{-n}^{-1}} p\left(z_{n}^{-1}\right) \log p\left(z_{n}^{-1}\right)\right) .
\end{aligned}
$$

With slight abuse of notation (by replacing the formal derivative with the derivative with respect to $\varepsilon$, we can define $\operatorname{High}_{\vec{N}}\left[p\left(z_{-n}^{0}\right)\right]=$ $\operatorname{High}_{\vec{N}}\left[p^{\varepsilon}\left(z_{-n}^{0}\right)\right]$. Similarly, for Low $\vec{N}\left[p\left(z_{-n}^{0}\right)\right]$, etc.), we have

$$
\begin{aligned}
& \left(p\left(z_{-n}^{0}\right) \log p\left(z_{-n}^{0}\right)\right)^{(\vec{N})}=\operatorname{High}_{\vec{N}}\left[p\left(z_{-n}^{0}\right)\right]+\operatorname{Low}_{\vec{N}}\left[p\left(z_{-n}^{0}\right)\right], \\
& \left(p\left(z_{-n}^{-1}\right) \log p\left(z_{-n}^{-1}\right)\right)^{(\vec{N})}=\operatorname{High}_{\vec{N}}\left[p\left(z_{-n}^{-1}\right)\right]+\operatorname{Low}_{\vec{N}}\left[p\left(z_{-n}^{-1}\right)\right] .
\end{aligned}
$$

Note that by Lemma 2.3, we have the first equation at the bottom of the page, and

$$
\begin{aligned}
\operatorname{High}_{\vec{N}}\left[p\left(z_{-n}^{-1}\right)\right] & \sum_{\lceil(|\vec{N}|+1) / 2\rceil \leq|\vec{i}| \leq|\vec{N}|} C_{\vec{N}}^{\vec{i}}\left(\log p\left(z_{-n}^{-1}\right)+1\right)^{(\vec{N}-\vec{i})} p\left(z_{-n}^{-1}\right)^{(\vec{\imath})} .
\end{aligned}
$$

Thus, we get the second array of equations at the bottom of the page. So the higher derivative part stabilizes at $\lceil(|\vec{N}|+1) / 2\rceil$, namely, for any $|\vec{n}| \geq\lceil(|\vec{N}|+1) / 2\rceil$

$$
\begin{aligned}
& \sum_{z_{-n}^{0}} \operatorname{High}_{\vec{N}}\left[p\left(z_{-n}^{0}\right)\right]-\sum_{z_{-n}^{-1}} \operatorname{High}_{\vec{N}}\left[p\left(z_{-n}^{-1}\right)\right]
\end{aligned}
$$

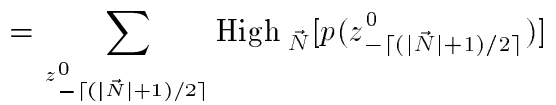

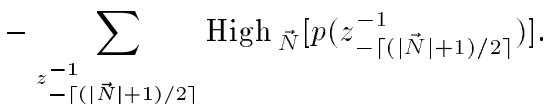

And by Lemma 2.4, we have

$$
\begin{aligned}
\operatorname{Low}_{\vec{N}}\left[p\left(z_{-n}^{0}\right)\right]= & \sum_{|\vec{i}| \leq\lceil(|\vec{N}|-1) / 2\rceil} r_{\vec{i}}\left[p\left(z_{0} \mid z_{-n}^{-1}\right)\right] p\left(z_{-n}^{-1}\right)^{(\vec{i})} \\
& \left.+\sum_{|\vec{i}| \leq\lceil(|\vec{N}|-1) / 2\rceil} s_{\vec{i}}^{\rightarrow}\left[p\left(z_{-n}^{-1}\right)\right)\right] p\left(z_{0} \mid z_{-n}^{-1}\right)^{(\vec{i})}
\end{aligned}
$$

$$
\operatorname{High}_{\vec{N}}\left[p\left(z_{-n}^{0}\right)\right]=\sum_{\lceil(|\vec{N}|+1) / 2\rceil \leq|i| \leq|\vec{N}|} C_{\vec{N}}^{\vec{i}}\left(\log p\left(z_{0} \mid z_{-n}^{-1}\right)+\log p\left(z_{-n}^{-1}\right)+1\right)^{(\vec{N}-\vec{i})} p\left(z_{-n}^{0}\right)^{(\vec{i})}
$$

$$
\begin{aligned}
\sum_{z_{-n}^{0}} \operatorname{High}_{\vec{N}}\left[p\left(z_{-n}^{0}\right)\right]-\sum_{z_{-n}^{-1}} \operatorname{High}_{\vec{N}}\left[p\left(z_{-n}^{-1}\right)\right] & =\sum_{z_{-n}^{0}\lceil(|\vec{N}|+1) / 2\rceil \leq|\vec{i}| \leq|\vec{N}|} \sum_{C_{\vec{N}}^{\vec{i}}\left(\log p\left(z_{0} \mid z_{-n}^{-1}\right)+\log p\left(z_{-n}^{-1}\right)-\log p\left(z_{-n}^{-1}\right)\right)^{(\vec{N}-\vec{i})} p\left(z_{-n}^{0}\right)^{(\vec{i})}} \sum \sum_{C_{\vec{N}}^{\vec{i}}\left(\log p\left(z_{0} \mid z_{-n}^{-1}\right)\right)^{(\vec{N}-\vec{i})} p\left(z_{-n}^{0}\right)^{(\vec{i})}} \sum^{z_{-n}^{0}\lceil(|\vec{N}|+1) / 2\rceil \leq|\vec{i}| \leq|\vec{N}|} \sum_{z_{-n}^{0}\lceil(|\vec{N}|+1) / 2\rceil \leq|\vec{i}| \leq|\vec{N}|} C_{\vec{N}}^{\vec{i}}\left(\log p\left(z_{0} \mid z_{-\Gamma(|\vec{N}|+1) / 2\rceil}^{-1}\right)\right)^{(\vec{N}-\vec{i})} p\left(z_{-\Gamma(|\vec{N}|+1) / 2\rceil}^{0}\right)^{(\vec{i})} .
\end{aligned}
$$


with $\left.s_{0}\left[p\left(z_{-n}^{-1}\right)\right)\right]=\operatorname{Low}_{\vec{N}}\left[p\left(z_{-n}^{-1}\right)\right]$. Thus

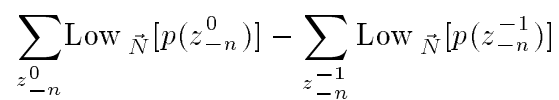

$$
\begin{aligned}
& =\sum_{z_{-n}^{0}|\vec{i}| \leq\lceil(|\vec{N}|-1) / 2\rceil} r_{\vec{i}}\left[p\left(z_{0} \mid z_{-n}^{-1}\right)\right] p\left(z_{-n}^{-1}\right)^{(\vec{i})} . \\
& =\sum_{z_{-n}^{0}|\vec{i}| \leq\lceil(|\vec{N}|-1) / 2\rceil} r_{\vec{i}}\left[p\left(z_{0} \mid z_{-\lceil(|\vec{N}|+1) / 2\rceil}^{-1}\right)\right] p\left(z_{-\lceil(|\vec{N}|+1) / 2\rceil}^{-1}\right)^{(\vec{i})} \text {. }
\end{aligned}
$$

Consequently, the lower derivative part stabilizes at $\lceil(|\vec{N}|+1) / 2\rceil$ as well, namely, for any $n \geq\lceil(|\vec{N}|+1) / 2\rceil$

$$
\begin{aligned}
& \sum_{z_{-n}^{0}} \operatorname{Low}_{\vec{N}}\left[p\left(z_{-n}^{0}\right)\right]-\sum_{\substack{z_{-n}^{-1} \\
z_{-\lceil(|\vec{N}|+1) / 2\rceil}}} \operatorname{Low}_{\vec{N}}\left[p\left(z_{-n}^{-1}\right)\right] \\
& \sum_{\substack{z_{-}^{-1} \\
-\lceil(|\vec{N}|+1) / 2\rceil}} \operatorname{Low}_{\vec{N}}\left[p\left(z_{-\lceil(|\vec{N}|+1) / 2\rceil}^{0}\right)\right]
\end{aligned}
$$

The theorem then follows.

Remark 2.6: For an irreducible stationary Markov chain $Y$ with probability transition matrix $\Delta$, let $Y^{-1}$ denote its reverse Markov chain. It is well known that the probability transition matrix of $Y^{-1}$ is $\operatorname{diag}\left(\pi_{1}^{-1}, \pi_{2}^{-1}, \ldots, \pi_{B}^{-1}\right) \Delta^{t} \operatorname{diag}\left(\pi_{1}, \pi_{2}, \ldots, \pi_{B}\right)$, where $\Delta^{t}$ denotes the transpose of $\Delta$ and $\left(\pi_{1}, \pi_{2}, \ldots, \pi_{B}\right)$ is the stationary vector of $Y$. Therefore, if $\Delta^{t}$ is a Black Hole case, the derivatives of $H\left(Z^{-1}\right)$ (here, $Z^{-1}$ is the reverse hidden Markov chain defined by $Z^{-1}=$ $\left.\Phi\left(Y^{-1}\right)\right)$ also stabilize. It then follows from $H(Z)=H\left(Z^{-1}\right)$ that the derivatives of $H(Z)$ also stabilize.

\section{BINARY MARKOV CHAINS CORRUPTED BY BINARY-SYMMETRIC NOISE}

In this section, we further study hidden Markov chains obtained by passing binary Markov chains through binary-symmetric channels with crossover probability $\varepsilon$ (described in the beginning of Section II). We take a concrete approach to study $H(Z)$, and we will "compute" $H^{\prime}(Z)$ in terms of Blackwell's measure.

Recall that the Markov chain is defined by a $2 \times 2$ stochastic matrix $\Pi=\left[\pi_{i j}\right]$. When $\operatorname{det}(\Pi)=0$, the rows of $\Pi$ are identical, and so $Y$ is an i.i.d. random sequence with distribution $\left(\pi_{00}, \pi_{01}\right)$. Thus, $Z$ is an i.i.d. random sequence with distribution $(\pi, 1-\pi)$ where $\pi=$ $\pi_{00}(1-\varepsilon)+\pi_{01} \varepsilon$. So

$$
H(Z)=-\pi \log \pi-(1-\pi) \log (1-\pi) .
$$

From now through the end of Section III-B, we assume the following:

- $\operatorname{det}(\Pi)>0-$ and -

- all $\pi_{i j}>0-$ and -

- $\varepsilon>0$.

We remark that the condition $\operatorname{det}(\Pi)>0$ is purely for convenience. Results in this section will hold with the condition $\operatorname{det}(\Pi)<0$ through similar arguments, unless specified otherwise.

The integral formula (1.1) expresses $H(Z)$ in terms of the measure $Q$ on the four-dimensional simplex; namely, $Q$ is the distribution of $p\left(\left(y_{0}, e_{0}\right) \mid z_{-\infty}^{0}\right)$. However, in the case under consideration, $H(Z)$ can be expressed as an integral on the real line [11], which we review as follows.
From the chain rule of probability theory

$$
\begin{aligned}
p\left(z_{1}^{i}, y_{i}\right)= & p\left(z_{1}^{i-1}, z_{i}, y_{i-1}=0, y_{i}\right)+p\left(z_{1}^{i-1}, z_{i}, y_{i-1}=1, y_{i}\right) \\
= & p\left(z_{i}, y_{i} \mid z_{1}^{i-1}, y_{i-1}=0\right) p\left(z_{1}^{i-1}, y_{i-1}=0\right) \\
& +p\left(z_{i}, y_{i} \mid z_{1}^{i-1}, y_{i-1}=1\right) p\left(z_{1}^{i-1}, y_{i-1}=1\right),
\end{aligned}
$$

and

$$
\begin{aligned}
& p\left(z_{i}, y_{i} \mid z_{1}^{i-1}, y_{i-1}=0\right) \\
& \quad=p\left(z_{1}^{i} \mid z_{1}^{i-1}, y_{i}, y_{i-1}=0\right) p\left(y_{i} \mid z_{1}^{i-1}, y_{i-1}=0\right) \\
& \quad=p\left(z_{i} \mid y_{i}\right) p\left(y_{i} \mid y_{i-1}=0\right)=p_{E}\left(e_{i}\right) p\left(y_{i} \mid y_{i-1}=0\right) .
\end{aligned}
$$

Let $a_{i}=p\left(z_{1}^{i}, y_{i}=0\right)$ and $b_{i}=p\left(z_{1}^{i}, y_{i}=1\right)$. The pair $\left(a_{i}, b_{i}\right)$ satisfies the following dynamical system:

$$
\left\{\begin{array}{l}
a_{i}=p_{E}\left(z_{i}\right) \pi_{00} a_{i-1}+p_{E}\left(z_{i}\right) \pi_{10} b_{i-1} \\
b_{i}=p_{E}\left(\bar{z}_{i}\right) \pi_{01} a_{i-1}+p_{E}\left(\bar{z}_{i}\right) \pi_{11} b_{i-1} .
\end{array}\right.
$$

Let $x_{i}=a_{i} / b_{i}$, we have a dynamical system with just one variable

$$
x_{i+1}=f_{z_{i+1}}\left(x_{i}\right) \text {, }
$$

where

starting with

$$
f_{z}(x)=\frac{p_{E}(z)}{p_{E}(\bar{z})} \frac{\pi_{00} x+\pi_{10}}{\pi_{01} x+\pi_{11}}, \quad z=0,1
$$

$$
x_{0}=\pi_{10} / \pi_{01} \text {. }
$$

We are interested in the invariant distribution of $x_{n}$, which is closely related to Blackwell's distribution of $p\left(\left(y_{0}, e_{0}\right) \mid z_{-\infty}^{0}\right)$. Now

$$
\begin{aligned}
p & \left(y_{i}=0 \mid z_{1}^{i-1}\right) \\
& =p\left(y_{i}=0, y_{i-1}=0 \mid z_{1}^{i-1}\right)+p\left(y_{i}=0, y_{i-1}=1 \mid z_{1}^{i-1}\right) \\
& =\pi_{00} p\left(y_{i-1}=0 \mid z_{1}^{i-1}\right)+\pi_{10} p\left(y_{i-1}=1 \mid z_{1}^{i-1}\right) \\
& =\pi_{00} \frac{a_{i-1}}{a_{i-1}+b_{i-1}}+\pi_{10} \frac{b_{i-1}}{a_{i-1}+b_{i-1}} \\
& =\pi_{00} \frac{x_{i-1}}{1+x_{i-1}}+\pi_{10} \frac{1}{1+x_{i-1}}
\end{aligned}
$$

Similarly, we have

$$
\begin{aligned}
& p\left(y_{i}=1 \mid z_{1}^{i-1}\right) \\
& \quad=p\left(y_{i}=1, y_{i-1}=0 \mid z_{1}^{i-1}\right)+p\left(y_{i}=1, y_{i-1}=1 \mid z_{1}^{i-1}\right) \\
& \quad=\pi_{01} \frac{x_{i-1}}{1+x_{i-1}}+\pi_{11} \frac{1}{1+x_{i-1}} .
\end{aligned}
$$

Further computation leads to

$$
\begin{aligned}
& p\left(z_{i}=0 \mid z_{1}^{i-1}\right) \\
& \quad=p\left(y_{i}=0, e_{i}=0 \mid z_{1}^{i-1}\right)+p\left(y_{i}=1, e_{i}=1 \mid z_{1}^{i-1}\right) \\
& \quad=p\left(e_{i}=0\right) p\left(y_{i}=0 \mid z_{1}^{i-1}\right)+p\left(e_{i}=1\right) p\left(y_{i}=1 \mid z_{1}^{i-1}\right) \\
& \quad=\left((1-\varepsilon) \pi_{00}+\varepsilon \pi_{01}\right) \frac{x_{i-1}}{1+x_{i-1}}+\left((1-\varepsilon) \pi_{10}+\varepsilon \pi_{11}\right) \frac{1}{1+x_{i-1}} \\
& \quad=r_{0}\left(x_{i-1}\right),
\end{aligned}
$$

where

$$
r_{0}(x)=\frac{\left((1-\varepsilon) \pi_{00}+\varepsilon \pi_{01}\right) x+\left((1-\varepsilon) \pi_{10}+\varepsilon \pi_{11}\right)}{x+1} .
$$

Similarly, we have

$$
\begin{aligned}
& p\left(z_{i}=1 \mid z_{1}^{i-1}\right) \\
& \quad=p\left(y_{i}=0, e_{i}=1 \mid z_{1}^{i-1}\right)+p\left(y_{i}=1, e_{i}=0 \mid z_{1}^{i-1}\right) \\
& \quad=p\left(e_{i}=1\right) p\left(y_{i}=0 \mid z_{1}^{i-1}\right)+p\left(e_{i}=0\right) p\left(y_{i}=1 \mid z_{1}^{i-1}\right) \\
& \quad=\left(\left(\varepsilon \pi_{00}+(1-\varepsilon) \pi_{01}\right) \frac{x_{i-1}}{1+x_{i-1}}+\left(\varepsilon \pi_{10}+(1-\varepsilon) \pi_{11}\right) \frac{1}{1+x_{i-1}}\right. \\
& \quad=r_{1}\left(x_{i-1}\right)
\end{aligned}
$$


where

$$
r_{1}(x)=\frac{\left(\varepsilon \pi_{00}+(1-\varepsilon) \pi_{01}\right) x+\left(\varepsilon \pi_{10}+(1-\varepsilon) \pi_{11}\right)}{x+1} .
$$

Now we write

$$
p\left(x_{i} \in E \mid x_{i-1}\right)=\sum_{\left\{a \mid f_{a}\left(x_{i-1}\right) \in E\right\}} p\left(z_{i}=a \mid x_{i-1}\right) .
$$

Note that

$$
\begin{aligned}
& p\left(z_{i}=0 \mid x_{i-1}\right)=p\left(z_{i}=0 \mid z_{1}^{i-1}\right)=r_{0}\left(x_{i-1}\right) \\
& p\left(z_{i}=1 \mid x_{i-1}\right)=p\left(z_{i}=1 \mid z_{1}^{i-1}\right)=r_{1}\left(x_{i-1}\right) .
\end{aligned}
$$

The analysis above leads to

$$
\begin{aligned}
p\left(x_{i} \in E\right) & \\
& =\int_{f_{0}^{-1}(E)} r_{0}\left(x_{i-1}\right) d p\left(x_{i-1}\right)+\int_{f_{1}^{-1}(E)} r_{1}\left(x_{i-1}\right) d p\left(x_{i-1}\right) .
\end{aligned}
$$

Abusing notation, we let $Q$ denote the limiting distribution of $x_{i}$ (the limiting distribution exists due to the martingale convergence theorem) and obtain

$$
Q(E)=\int_{f_{0}^{-1}(E)} r_{0}(x) d Q(x)+\int_{f_{1}^{-1}(E)} r_{1}(x) d Q(x) .
$$

We may now compute the entropy rate of $Z_{i}$ in terms of $Q$. Note that

$$
\begin{aligned}
& E\left(\log p\left(z_{i} \mid z_{1}^{i-1}\right)\right) \\
& =E\left(p\left(z_{i}=0 \mid z_{1}^{i-1}\right) \log p\left(z_{i}=0 \mid z_{1}^{i-1}\right)\right) \\
& \left.\quad+p\left(z_{i}=1 \mid z_{1}^{i-1}\right) \log p\left(z_{i}=1 \mid z_{1}^{i-1}\right)\right) \\
& =E\left(r_{0}\left(x_{i-1}\right) \log r_{0}\left(x_{i-1}\right)+r_{1}\left(x_{i-1}\right) \log r_{1}\left(x_{i-1}\right)\right) .
\end{aligned}
$$

Thus, (1.1) becomes

$$
H(Z)=-\int\left(r_{0}(x) \log r_{0}(x)+r_{1}(x) \log r_{1}(x)\right) d Q(x) .
$$

\section{A. Properties of $Q$}

Since $\operatorname{det}(\Pi)>0, f_{0}$ and $f_{1}$ are increasing continuous functions bounded from above, and $f_{0}(0)$ and $f_{1}(0)$ are positive; therefore, they each have a unique positive fixed point $p_{0}$ and $p_{1}$. Since $f_{1}$ is dominated by $f_{0}$, we conclude $p_{1} \leq p_{0}$. Let

- $I$ denote the interval $\left[p_{1}, p_{0}\right]-$ and -

- $L=\bigcup_{n=1}^{\infty} L_{n}$ where

$$
L_{n}=\left\{f_{i_{1}} \circ f_{i_{2}} \cdots \circ f_{i_{n}}\left(p_{j}\right) \mid i_{1}, i_{2}, \ldots, i_{n} \in\{0,1\}, j=0,1\right\} .
$$

Let $I_{i_{1} i_{2} \cdots i_{n}}$ denote $f_{i_{n}} \circ f_{i_{n-1}} \circ \cdots \circ f_{i_{1}}(I)$, and $p_{i_{1} i_{2} \cdots i_{n}}$ denote $p\left(z_{1}=i_{1}, z_{2}=i_{2}, \ldots, z_{n}=i_{n}\right)$. The support of a probability measure $Q$, denoted $\operatorname{supp}(Q)$, is defined as the smallest closed subset with measure one.

Theorem 3.1: $\operatorname{supp}(Q)=\bar{L}$.

Proof: First, by straightforward computation, one can check that $f_{0}^{\prime}\left(p_{0}\right)$ and $f_{1}^{\prime}\left(p_{1}\right)$ are both less than 1 . Thus, $p_{0}$ and $p_{1}$ are attracting fixed points. Since $p_{i}$ is the unique positive fixed point of $f_{i}$, it follows that the entire positive half of the real line is in the domain of attraction of each $f_{i}$, i.e., for any $p>0, f_{i}^{(n)}(p)$ approaches $p_{i}$ (here the superscript $^{(n)}$ denotes the composition of $n$ copies of the function).

We claim that both $p_{0}$ and $p_{1}$ are in $\operatorname{supp}(Q)$. If $p_{0}$ is not in the support, then there is a neighborhood $I_{p_{0}}$ containing $p_{0}$ with $Q$-measure 0 . For any point $p>0$, for some $n, f_{0}^{(n)}(p) \in I_{p_{0}}$. Thus, by (3.10) there is a neighborhood of $p$ with $Q$-measure 0 . It follows that $Q([0, \infty))=0$. On the other hand, $Q$ is the limiting distribution of $x_{i}>0$ and so $Q([0, \infty))=1$. This contradiction shows that $p_{0} \in \operatorname{supp}(Q)$. Similarly, $p_{1} \in \operatorname{supp}(Q)$.

By (3.10), we deduce

$$
f_{i}(\operatorname{supp}(Q)) \subseteq \operatorname{supp}(Q) .
$$

It follows that $L \subseteq \operatorname{supp}(Q)$. Thus, $\bar{L} \subseteq \operatorname{supp}(Q)$.

Since $f_{i}((0, \infty))$ is contained in a compact set, we may assume $f_{i}$ is a contraction mapping (otherwise, compose $f_{0}$ or $f_{1}$ enough many times to make the composite mapping a contraction as we argued in [4]). In this case, the set of accumulation points of $\left\{f_{i_{n}} \circ f_{i_{n-1}} \cdots \circ\right.$ $\left.f_{i_{1}}(p) \mid i_{1}, i_{2}, \ldots, i_{n} \in\{0,1\}, p>0\right\}$ does not depend on $p$. Since any point in $\operatorname{supp}(Q)$ has to be an accumulation point of $\left\{f_{i_{n}} \circ f_{i_{n-1}} \cdots \circ\right.$ $\left.f_{i_{1}}\left(\pi_{10} / \pi_{01}\right) \mid i_{1}, i_{2}, \ldots, i_{n} \in\{0,1\}\right\}$, it has to be an accumulation point of $L$ as well, which implies $\operatorname{supp}(Q) \subseteq \bar{L}$.

It is easy to see the following.

Lemma 3.2: The following statements are equivalent.

1. $f_{0}(I) \cup f_{1}(I) \varsubsetneqq I$.

2. $f_{0}(I) \cap f_{1}(I) \stackrel{\neq}{=} \phi$

3. $f_{1}\left(p_{0}\right)<f_{0}\left(p_{1}\right)$.

Theorem 3.3: $\operatorname{supp}(Q)$ is either a Cantor set or a closed interval. Specifically

1. $\operatorname{supp}(Q)$ is a Cantor set if $f_{0}(I) \cup f_{1}(I) \varsubsetneqq I$;

2. $\operatorname{supp}(Q)=I$ if equivalently $f_{0}(I) \cup f_{1}(I)=I$.

Proof: Suppose that $f_{0}(I) \cup f_{1}(I) \varsubsetneqq I$. If $\left(i_{1}, i_{2}, \ldots, i_{n}\right) \neq$ $\left(j_{1}, j_{2}, \ldots, j_{n}\right)$, then

Define

$$
I_{i_{1} i_{2} \cdots i_{n}} \cap I_{j_{1} j_{2} \cdots j_{n}}=\phi .
$$

$$
I_{\langle n\rangle}=\bigcup_{i_{1}, i_{2}, \ldots, i_{n}} I_{i_{1} i_{2} \cdots i_{n}} .
$$

Alternatively we can construct $I_{\langle n\rangle}$ as follows: let

$$
I^{d}=\left(f_{1}\left(p_{0}\right), f_{0}\left(p_{1}\right)\right)
$$

then

$$
I_{\langle n+1\rangle}=I_{\langle n\rangle} \backslash \bigcup_{i_{1}, i_{2}, \ldots, i_{n}} f_{i_{n}} \circ f_{i_{n-1}} \circ \cdots \circ f_{i_{1}}\left(I^{d}\right) .
$$

Let $I_{\langle\infty\rangle}=\bigcap_{n=1}^{\infty} I_{\langle n\rangle}$. It follows from the way it is constructed that $I_{\infty}$ is a Cantor set (think of $I^{d}$ as a "deleted" interval), and $\bar{L}=I_{\langle\infty\rangle}$. Thus, by Theorem $3.1, \operatorname{supp}(Q)=\bar{L}$ is a Cantor set.

Suppose $f_{0}(I) \cup f_{1}(I)=I$. In this case, for any point $p \in I$, and for all $n$, there exists $i_{1}, i_{2}, \ldots, i_{n}$ such that

$$
p \in I_{i_{1} i_{2} \cdots i_{n}} .
$$

From the fact that $f_{0}$ and $f_{1}$ are both contraction mappings (again, otherwise compose $f_{0}$ or $f_{1}$ enough many times to make the composite mapping a contraction as we argued in [4]), we deduce that the length of $I_{i_{1} i_{2} \cdots i_{n}}$ is exponentially decreasing with respect to $n$. It follows that $L$ is dense in $I$, and therefore, $\operatorname{supp}(Q)=\bar{L}=I$.

Theorem 3.4: $Q$ is a continuous measure, namely for any point $p \in$ $\operatorname{supp}(Q)$, and for any $\eta>0$, there exists an interval $I_{p}$ containing $p$ with $Q\left(I_{p}\right)<\eta$ (or equivalently $Q$ has no point mass).

Proof: Assume that there exists $p \in I$ such that for any interval containing $p, Q\left(I_{p}\right)>\eta_{0}$, where $\eta_{0}$ is a positive constant. Let $\xi=$ $\max \left\{r_{0}(x), r_{1}(x): x \in I\right\}$. One checks that $0<\xi<1$. By (3.10), we have

$$
\frac{1}{\xi} Q\left(I_{p}\right) \leq Q\left(f_{0}^{-1}\left(I_{p}\right)\right)+Q\left(f_{1}^{-1}\left(I_{p}\right)\right)
$$


Iterating, we obtain

$$
\left(\frac{1}{\xi}\right)^{n} \eta_{0} \leq \sum_{i_{1}, i_{2}, \ldots, i_{n}} Q\left(f_{i_{1}}^{-1} \circ f_{i_{2}}^{-1} \circ \cdots \circ f_{i_{n}}^{-1}\left(I_{p}\right)\right) .
$$

For fixed $n$, if we choose $I_{p}$ small enough, then

$$
f_{i_{1}}^{-1} \circ f_{i_{2}}^{-1} \circ \cdots \circ f_{i_{n}}^{-1}\left(I_{p}\right) \cap f_{j_{1}}^{-1} \circ f_{j_{2}}^{-1} \circ \cdots \circ f_{j_{n}}^{-1}\left(I_{p}\right)=\phi
$$

for $\left(i_{1}, i_{2}, \ldots, i_{n}\right) \neq\left(j_{1}, j_{2}, \ldots, j_{n}\right)$. It follows in this case that

$$
Q(I) \geq \sum_{i_{1}, i_{2}, \ldots, i_{n}} Q\left(\left(f_{i_{1}}^{-1} \circ f_{i_{2}}^{-1} \circ \cdots \circ f_{i_{n}}^{-1}\left(I_{p}\right)\right) \geq\left(\frac{1}{\xi}\right)^{n} \eta_{0} .\right.
$$

Therefore, for large $n$, we deduce

$$
Q(I)>1
$$

which contradicts the fact that $Q$ is a probability measure.

By virtue of Lemma 3.2, it makes sense to refer to Case 1 in Theorem 3.3 as the nonoverlapping case. We now focus on this case. Note that this is the case whenever $\varepsilon$ is sufficiently small; also, it turns out that for some values of $\pi_{i j}$ 's, the nonoverlapping case holds for all $\varepsilon$.

Starting with $x_{0}=\pi_{10} / \pi_{01}$, and iterating according to $x_{n}=f_{z_{n}}\left(\varepsilon, x_{n-1}\right)$, each word $z=z_{1}, z_{2}, \ldots, z_{n}$ determines a point $x_{n}=x_{n}(z)$ with probability $p\left(z_{1}, z_{2}, \ldots, z_{n}\right)$. In the nonoverlapping case, the map $z \mapsto x_{n}$ is one-to-one. We order the distinct points $\left\{x_{n}\right\}$ from left to right as

$$
x_{n, 1}, x_{n, 2}, \ldots, x_{n, 2} n
$$

with the associated probabilities

$$
p_{n, 1}, p_{n, 2}, \ldots, p_{n, 2} \text {. }
$$

This defines a sequence of distribution $Q_{n}$ which converge weakly to $Q$. In particular, by the continuity of $Q, Q_{n}(J) \rightarrow Q(J)$ for any interval $J$.

Theorem 3.5: In the nonoverlapping case

$$
Q\left(I_{i_{1} i_{2} \cdots i_{n}}\right)=Q_{n}\left(I_{i_{1} i_{2} \cdots i_{n}}\right)=p_{i_{1} i_{2} \cdots i_{n}}
$$

Proof: We have

$$
Q_{n}\left(I_{i_{1} i_{2} \cdots i_{n}}\right)=p\left(z_{1}=i_{1}, z_{2}=i_{2}, \ldots, z_{n}=i_{n}\right) .
$$

Furthermore

$$
\begin{aligned}
Q_{n+1}\left(I_{i_{1} i_{2} \cdots i_{n}}\right)= & Q_{n+1}\left(I_{0 i_{1} i_{2} \cdots i_{n}}\right)+Q_{n+1}\left(I_{1 i_{1} i_{2} \cdots i_{n}}\right) \\
= & p\left(z_{0}=0, z_{1}=i_{1}, z_{2}=i_{2}, \ldots, z_{n}=i_{n}\right) \\
& +p\left(z_{0}=1, z_{1}=i_{1}, z_{2}=i_{2}, \ldots, z_{n}=i_{n}\right) \\
= & p\left(z_{1}=i_{1}, z_{2}=i_{2}, \ldots, z_{n}=i_{n}\right) .
\end{aligned}
$$

Iterating one shows that for $m \geq n$

$$
Q_{m}\left(I_{i_{1} i_{2} \cdots i_{n}}\right)=Q_{n}\left(I_{i_{1} i_{2} \cdots i_{n}}\right)=p_{i_{1} i_{2} \cdots i_{n}}
$$

By the continuity of $Q$ (Theorem 3.4)

$$
Q\left(I_{i_{1} i_{2} \cdots i_{n}}\right)=p_{i_{1} i_{2} \cdots i_{n}}
$$

From this, as in [11], [12] we can derive bounds for the entropy rate. Let

$$
r(x)=-\left(r_{0}(x) \log r_{0}(x)+r_{1}(x) \log r_{1}(x)\right) .
$$

Using (3.11) and Theorem 3.5, we obtain the following.

Theorem 3.6: In the nonoverlapping case

$$
\sum_{i_{1} i_{2} \cdots i_{n}} r_{i_{1} i_{2} \cdots i_{n}}^{m} p_{i_{1} i_{2} \cdots i_{n}} \leq H(Z) \leq \sum_{i_{1} i_{2} \cdots i_{n}} r_{i_{1} i_{2} \cdots i_{n}}^{M} p_{i_{1} i_{2} \cdots i_{n}}
$$

where

and

$$
r_{i_{1} i_{2} \cdots i_{n}}^{m}=\min _{x \in I_{i_{1} i_{2} \cdots i_{n}}} r(x)
$$

$$
r_{i_{1} i_{2} \cdots i_{n}}^{M}=\max _{x \in I_{i_{1} i_{2} \cdots i_{n}}} r(x) .
$$

Proof: This follows immediately from the formula for the entropy rate $H(Z)(3.11)$.

\section{B. Computation of the First Derivative in Nonoerlapping Case}

To emphasize the dependence on $\varepsilon$, we write $p_{n, i}(\varepsilon)=p_{n, i}$, $x_{n, i}(\varepsilon)=x_{n, i}, p_{0}(\varepsilon)=p_{0}, p_{1}(\varepsilon)=p_{1}$, and $Q_{n}(\varepsilon)=Q_{n}$. Let $F_{n}(\varepsilon, x)$ denote the cumulative distribution function of $Q_{n}(\varepsilon)$. Let $H_{n}^{\varepsilon}(Z)$ be the finite approximation to $H^{\varepsilon}(Z)$. It can be easily checked that

$$
H_{n}^{\varepsilon}(Z)=\int_{I} r(\varepsilon, x) d Q_{n}(\varepsilon)
$$

and we can rewrite (3.11) as

$$
H^{\varepsilon}(Z)=\int_{I} r(\varepsilon, x) d Q(\varepsilon) .
$$

In Theorem 3.7, we express the derivative of the entropy rate, with respect to $\varepsilon$, as the sum of four terms which have meaningful interpretations. Essentially, we are differentiating $H^{\varepsilon}(Z)$ with respect to $\varepsilon$ under the integral sign, but care must be taken since $Q(\varepsilon)$ is generally singular and varies with $\varepsilon$.

Rewriting this using the Riemann-Stieltjes integral and applying integration by parts, we obtain

$$
\begin{aligned}
H_{n}^{\varepsilon}(Z) & =\int_{I} r(\varepsilon, x) d F_{n}(\varepsilon, x) \\
& =\left.F_{n}(\varepsilon, x) r(\varepsilon, x)\right|_{p_{1}(\varepsilon)} ^{p_{0}(\varepsilon)}-\int_{I} F_{n}(\varepsilon, x) g(\varepsilon, x) d x
\end{aligned}
$$

where $g(\varepsilon, x)=\frac{\partial r(\varepsilon, x)}{\partial x}$.

From now on ' denotes the derivative with respect to $\varepsilon$. Now

$$
H_{n}^{\varepsilon}(Z)^{\prime}=r\left(\varepsilon, p_{0}(\varepsilon)\right)^{\prime}-D_{n}(\varepsilon)
$$

where

$$
D_{n}(\varepsilon)=\lim _{h \rightarrow 0} \frac{\int_{I} F_{n}(\varepsilon+h, x) g(\varepsilon+h, x) d x-\int_{I} F_{n}(\varepsilon, x) g(\varepsilon, x) d x}{h} .
$$

We can decompose $D_{n}(\varepsilon)$ into two terms

and

$$
\begin{aligned}
& D_{n}(\varepsilon)=D_{n}^{1}(\varepsilon)+D_{n}^{2}(\varepsilon), \\
& D_{n}^{1}(\varepsilon)=\lim _{h \rightarrow 0} \int_{I} \frac{F_{n}(\varepsilon+h, x)-F_{n}(\varepsilon, x)}{h} g(\varepsilon, x) d x,
\end{aligned}
$$

$$
D_{n}^{2}(\varepsilon)=\int_{I} F_{n}(\varepsilon, x) g^{\prime}(\varepsilon, x) d x
$$


In order to compute $D_{n}^{1}(\varepsilon)$, we partition $I$ into two pieces: 1) small intervals $\left(x_{n, i}(\varepsilon), x_{n, i}(\varepsilon+h)\right)$ and 2$)$ the complement of the union of these neighborhoods, to yield

$$
\begin{aligned}
D_{n}^{1}(\varepsilon)= & \lim _{h \rightarrow 0} \int_{I} \frac{F_{n}(\varepsilon+h, x)-F_{n}(\varepsilon, x)}{h} g(\varepsilon, x) d x= \\
& -\sum_{i} p_{n, i}(\varepsilon) x_{n, i}(\varepsilon)^{\prime} g\left(\varepsilon, x_{n, i}\right)(\varepsilon)+\int_{I} F_{n}^{\prime}(\varepsilon, x) g(\varepsilon, x) d x .
\end{aligned}
$$

Combining the foregoing expressions, we arrive at an expression for $H_{n}^{\varepsilon}(Z)^{\prime}$

$$
\begin{aligned}
H_{n}^{\varepsilon}(Z)^{\prime}=r\left(\varepsilon, p_{0}(\varepsilon)\right)^{\prime} & +\sum_{i} p_{n, i}(\varepsilon) x_{n, i}^{\prime}(\varepsilon) g\left(\varepsilon, x_{n, i}(\varepsilon)\right) \\
& -\int_{I} F_{n}^{\prime}(\varepsilon, x) g(\varepsilon, x) d x-\int_{I} F_{n}(\varepsilon, x) g^{\prime}(\varepsilon, x) d x .
\end{aligned}
$$

Write $H^{\varepsilon}(Z)=H(Z), Q(\varepsilon)=Q$, and let $F(\varepsilon, x)$ be the cumulative distribution function of $Q(\varepsilon)$.

We then show that $H_{n}^{\varepsilon}(Z)$ converges uniformly to $H^{\varepsilon}(Z)$ and $H_{n}^{\varepsilon}(Z)^{\prime}$ converges uniformly to some function; it follows that this function is $H^{\varepsilon}(Z)^{\prime}$. This requires showing that the integrands in the second and third terms of the previous expression converge to well-defined functions.

We think of the $x_{n, i}(\varepsilon)$ as locations of point masses. So, we can think of $x_{n, i}(\varepsilon)^{\prime}$ as an instantaneous location change.

1. Second Term, Instantaneous Location Change (See Appendix C): For $x \in \operatorname{supp}(Q(\varepsilon))$ and any sequence of points $x_{n_{1}, i_{1}}(\varepsilon), x_{n_{2}, i_{2}}(\varepsilon), \ldots$ approaching $x$,

$$
K_{1}(\varepsilon, x)=\lim _{j \rightarrow \infty} x_{n_{j}, i_{j}}^{\prime}(\varepsilon)
$$

is a well-defined continuous function.

2. Third Term, Instantaneous Probability Change (See Appendix D): Recall that $\operatorname{supp}(Q(\varepsilon))$ is a Cantor set defined by a collection of "deleted" intervals: namely, $I^{d} \equiv\left(f_{0}\left(p_{1}\right), f_{1}\left(p_{0}\right)\right)$, and all intervals of the form $f_{i_{1}} \circ f_{i_{2}} \circ \cdots \circ f_{i_{n}}\left(I^{d}\right)$ (called deleted intervals on level $n$ ). For $x$ belonging to a deleted interval on level $n$, define $K_{2}(\varepsilon, x)=F_{n}^{\prime}(\varepsilon, x)$. Since the union of deleted intervals is dense in $I$, we can extend $K_{2}(\varepsilon, x)$ to a function on all $x \in I$, and we show that $K_{2}(\varepsilon, x)$ is a well-defined continuous function.

Using the boundedness of the instantaneous location change and probability change (established in Appendix A and Appendix B) and the Arzela-Ascoli theorem (note that Appendix C and Appendix D imply pointwise convergence of $H_{n}^{\varepsilon}(Z)^{\prime}$ and Appendix A and Appendix B imply equicontinuity of $\left.H_{n}^{\varepsilon}(Z)^{\prime}\right)$, we obtain uniform convergence of $H_{n}^{\varepsilon}(Z)^{\prime}$ to $H^{\varepsilon}(Z)^{\prime}$, which gives the result:

Theorem 3.7: In the nonoverlapping case

$$
\begin{aligned}
H^{\varepsilon}(Z)^{\prime}=r\left(\varepsilon, p_{0}(\varepsilon)\right)^{\prime} & +\int_{\operatorname{supp}(Q(\varepsilon))} K_{1}(\varepsilon, x) g(\varepsilon, x) d F(\varepsilon, x) \\
& -\int_{I} K_{2}(\varepsilon, x) g(\varepsilon, x) d x-\int_{I} F(\varepsilon, x) g^{\prime}(\varepsilon, x) d x .
\end{aligned}
$$

Note that the second term in this expression is a weighted mean of the instantaneous location change and the third term in this expression is a weighted mean of the instantaneous probability change.

Remark 3.8: Using the same technique, we can give a similar formula for the derivative of $H^{\varepsilon}(Z)$ with respect to $\pi_{i j}$ 's when $\varepsilon>0$. We can also give such formulas for higher derivatives in a similar way.
Remark 3.9: The techniques in this section can be applied to give an expression for the derivative of the entropy rate in the special overlapping case where $f_{0}\left(p_{1}\right)=f_{1}\left(p_{0}\right)$.

\section{Derivatives in Other Cases}

1. If any two of the $\pi_{i j}$ 's are equal to 0 , then

$$
H^{\varepsilon}(Z)=-\varepsilon \log \varepsilon-(1-\varepsilon) \log (1-\varepsilon)
$$

$H^{\varepsilon}(Z)$ is not differentiable with respect to $\varepsilon$ at $\varepsilon=0$.

2. Of more interest, it was shown in [12] that $H(Z)$ is not differentiable with respect to $\varepsilon$ at $\varepsilon=0$ when exactly one of the $\pi_{i j}$ 's is equal to 0 .

3. Consider the case that $\varepsilon=0$ and all the $\pi_{i j}$ 's are positive. As discussed in Example 4.1 of [4], the entropy rate is analytic as a function of $\varepsilon$ and $\pi_{i j}$ 's.

In [6] (and more generally in [16], [17]), an explicit formula was given for $H^{\prime}(Z)$ at $\varepsilon=0$ in this case. We briefly indicate how this is related to our results in Section III-B.

Instead of considering the dynamics of $x_{n}$ on the real line, we consider those of $\left(a_{n}, b_{n}\right)$ on the one-dimensional simplex

$$
W=\left\{\left(w_{1}, w_{2}\right): w_{1}+w_{2}=1, w_{i} \geq 0\right\} .
$$

Let $Q$ denote the limiting distribution of $\left(a_{n}, b_{n}\right)$ on $W$, the entropy $H(Z)$ can be computed as follows:

$$
H(Z)=\int_{W}-\left(r_{0}(w) \log r_{0}(w)+r_{1}(w) \log r_{1}(w)\right) d Q
$$

where

$$
\begin{aligned}
& r_{0}(w)=\left((1-\varepsilon) \pi_{00}+\varepsilon \pi_{01}\right) w_{1}+\left((1-\varepsilon) \pi_{10}+\varepsilon \pi_{11}\right) w_{2} \\
& r_{1}(w)=\left(\left(\varepsilon \pi_{00}+(1-\varepsilon) \pi_{01}\right) w_{1}+\left(\varepsilon \pi_{10}+(1-\varepsilon) \pi_{11}\right) w_{2} .\right.
\end{aligned}
$$

In order to calculate the derivative, we split the region of integration into two disjoint parts $W=W^{0} \cup W^{1}$ with

$$
\begin{aligned}
& W^{0}=\{t(0,1)+(1-t)(1 / 2,1 / 2): 0 \leq t \leq 1\} \\
& W^{1}=\{t(1 / 2,1 / 2)+(1-t)(1,0): 0 \leq t \leq 1\} .
\end{aligned}
$$

Let $r(w)=-\left(r_{0}(w) \log r_{0}(w)+r_{1}(w) \log r_{1}(w)\right)$ and $H^{i}(Z)=\int_{W^{i}} r(w) d Q$, then

$$
H(Z)=H^{0}(Z)+H^{1}(Z) .
$$

For $W^{0}$, we represent every point $\left(w_{1}, w_{2}\right)$ using the coordinate $w_{1} / w_{2}$. For $W^{1}$, we represent every point $\left(w_{1}, w_{2}\right)$ using the coordinate $w_{2} / w_{1}$. Computation shows that $H_{n}^{\varepsilon}(Z)$ uniformly converge to $H^{\varepsilon}(Z)$ on $[0,1 / 2]$. Note that expressions in Theorem 3.7 are not computable for $\varepsilon>0$, however, we can apply similar uniform convergence ideas in each of these regions to recover the formula given in [6] for $\varepsilon=0$.

4. (Low signal-to-noise ratio (SNR) regime, $\varepsilon=1 / 2$ ) In Corollary 6 of [11], it was shown that in the symmetric case (i.e., $\pi_{01}=$ $\left.\pi_{10}\right)$, the entropy rate approaches zero at rate $(1 / 2-\varepsilon)^{4}$ as $\varepsilon$ approaches $1 / 2$. It can be shown that the entropy rates at $\varepsilon$ and $1-\varepsilon$ are the same, and so all odd-order derivatives vanish at $\varepsilon=$ $1 / 2$. It follows that this result of [11] is equivalent to the statement that in the symmetric case $\left.H^{\prime \prime}(Z)\right|_{\varepsilon=1 / 2}=0$. We generalize this result to the nonsymmetric case as follows:

$$
\left.H^{\prime \prime}(Z)\right|_{\varepsilon=1 / 2}=-4\left(\frac{\pi_{10}-\pi_{01}}{\pi_{10}+\pi_{01}}\right)^{2} .
$$

For more details, see Appendix E. 


\section{APPENDIX A}

\section{Proof of Boundedness of InstantanEOUS Location CHANGE}

Claim: For any fix $0<\eta<1 / 2, x_{n, i}^{(k)}(\varepsilon) \leq C_{1}(k, \eta), \eta \leq \varepsilon \leq$ $1 / 2, C_{1}$ is a positive constant only depending on $k, \eta$.

Proof: We only prove the case when $k=1$. Consider the iteration

$$
x_{n+1}=f_{z_{n+1}}\left(\varepsilon, x_{n}\right) \text {. }
$$

Taking the derivative with respect to $\varepsilon$, we obtain

$$
x_{n+1}^{\prime}=\frac{\partial f_{z_{n+1}}}{\partial \varepsilon}\left(\varepsilon, x_{n}\right)+\frac{\partial f_{z_{n+1}}}{\partial x}\left(\varepsilon, x_{n}\right) x_{n}^{\prime} .
$$

Note that $\frac{\partial f_{z_{n+1}}}{\partial \varepsilon}\left(\varepsilon, x_{n}\right)$ is uniformly bounded by a constant and $\frac{\partial f_{z_{n+1}}}{\partial x}\left(\varepsilon, x_{n}\right)$ is bounded by $\rho$ with $0<\rho<1$, we conclude $x_{n}^{\prime}$ is uniformly bounded too.

\section{APPENDIX B}

\section{Proof of Boundedness of Instantaneous Probability Change}

Claim: For $x \notin\left\{x_{n, i}\right\}$ and $0 \leq \varepsilon \leq 1 / 2, F_{n}^{(k)}(\varepsilon, x) \leq C_{2}(k)$, where $C_{2}$ is a positive constant only depending on $k$.

Proof: We only prove the case when $k=1$. For $x$ with $x_{n, 2 i}<$ $x<x_{n, 2 i+1}$, we have $F_{n}(\varepsilon, x)=F_{n-1}(\varepsilon, x)$, and consequently

$$
\frac{\partial F_{n}(\varepsilon, x)}{\partial \varepsilon}=\frac{\partial F_{n-1}(\varepsilon, x)}{\partial \varepsilon} .
$$

For $x$ with $x_{n, 2 i-1}<x<x_{n, 2 i}, \frac{\partial F_{n}(\varepsilon, x)}{\partial \varepsilon}-\frac{\partial F_{n-1}(\varepsilon, x)}{\partial \varepsilon}$ is bounded by $C \rho_{1}^{n}$, here $C$ is a positive constant and $0<\rho_{1}<1$ (see proof that $K_{2}$ is well defined in Appendix D). Therefore, we conclude that the instantaneous probability change is uniformly bounded.

\section{APPENDIX C \\ Proof That $K_{1}$ Is WeLl DEFINED}

Proof: We need to prove that if two points $x_{n_{k}, i_{k}}$ and $x_{n_{l}, i_{l}}$ are close, then $x_{n_{k}, i_{k}}^{\prime}$ and $x_{n_{l}, i_{l}}^{\prime}$ are also close. Note that for nonoverlapping case, if $x_{n_{k}, i_{k}}$ and $x_{n_{l}, i_{l}}$ are very close, their corresponding symbolic sequences must share a long common tail. We shall prove that the asymptotical dynamics of $x_{n}$ does not depend on the starting point as long as they have the same common long tail. Without loss of generality, we assume that $z, \hat{z}$ have common tail $z_{1}, z_{2}, \ldots, z_{n}$. In this case, the two dynamical systems start with different values $x_{0}, \hat{x}_{0}$ along the same path. Now the two iterations produce

$$
\begin{aligned}
x_{n+1}^{\prime} & =\frac{\partial f_{z_{n+1}}}{\partial \varepsilon}\left(\varepsilon, x_{n}\right)+\frac{\partial f_{z_{n+1}}}{\partial x}\left(\varepsilon, x_{n}\right) x_{n}^{\prime} \\
\hat{x}_{n+1}^{\prime} & =\frac{\partial f_{z_{n+1}}}{\partial \varepsilon}\left(\varepsilon, \hat{x}_{n}\right)+\frac{\partial f_{z_{n+1}}}{\partial x}\left(\varepsilon, \hat{x}_{n}\right) \hat{x}_{n}^{\prime} .
\end{aligned}
$$

Taking the difference, we have

$$
\begin{aligned}
x_{n+1}^{\prime}-\hat{x}_{n+1}^{\prime}= & \frac{\partial f_{z_{n+1}}}{\partial \varepsilon}\left(\varepsilon, x_{n}\right)-\frac{\partial f_{z_{n+1}}}{\partial \varepsilon}\left(\varepsilon, \hat{x}_{n}\right) \\
& +\frac{\partial f_{z_{n+1}}}{\partial x}\left(\varepsilon, x_{n}\right) x_{n}^{\prime}-\frac{\partial f_{z_{n+1}}}{\partial x}\left(\varepsilon, \hat{x}_{n}\right) \hat{x}_{n}^{\prime} \\
= & \frac{\partial f_{z_{n+1}}}{\partial \varepsilon}\left(\varepsilon, x_{n}\right)-\frac{\partial f_{z_{n+1}}}{\partial \varepsilon}\left(\varepsilon, \hat{x}_{n}\right) \\
& +\frac{\partial f_{z_{n+1}}}{\partial x}\left(\varepsilon, x_{n}\right) x_{n}^{\prime}-\frac{\partial f_{z_{n+1}}}{\partial x}\left(\varepsilon, \hat{x}_{n}\right) x_{n}^{\prime} \\
& +\frac{\partial f_{z_{n+1}}}{\partial x}\left(\varepsilon, \hat{x}_{n}\right) x_{n}^{\prime}-\frac{\partial f_{z_{n+1}}}{\partial x}\left(\varepsilon, \hat{x}_{n}\right) \hat{x}_{n}^{\prime} .
\end{aligned}
$$

Since

- when $n \rightarrow \infty, x_{n}$ and $\hat{x}_{n}$ are getting close uniformly with respect to $\varepsilon-$ and -

- $\frac{\partial f_{i}}{\partial \varepsilon}(\varepsilon, \cdot)$ and $\frac{\partial f_{i}}{\partial x}(\varepsilon, \cdot)(i=0,1)$ are Lipschitz - and -

- $f_{i}(\varepsilon, \cdot)(i=0,1)$ are $\rho$-contraction mappings,

we conclude that $x_{n}^{\prime}$ and $\hat{x}_{n}^{\prime}$ are very close uniformly with respect to $\varepsilon$. The well definedness of $K_{1}$ then follows.

\section{APPENDIX D}

\section{PRoOF That $K_{2}$ Is Well DeFINED}

Proof: Every deleted interval corresponds to a finite sequence of binary digits and $K_{2}$ is well defined on these intervals. We order the deleted intervals on level $n$ from left to right

$$
I_{n, 1}^{d}, I_{n, 2}^{d}, \ldots, I_{n, 2}^{d}{ }^{n-1} .
$$

We need to prove that if two deleted intervals $I_{m, i}^{d}, I_{n, j}^{d}$ are close, then $F_{m}\left(\varepsilon, I_{m, i}^{d}\right)$ (which is defined as $F_{m}(\varepsilon, x)$ with $\left.x \in I_{m, i}^{d}\right)$ and $F_{m}\left(\varepsilon, I_{m, i}^{d}\right)$ are close. Assume $m \leq n$, then the points $x_{n, k}$ 's in between $I_{m, i}^{d}$ and $I_{n, j}^{d}$ must have a long common tail. Suppose that the common tail is the path $z_{1}, z_{2}, \ldots, z_{n}$, let $q_{i}$ denote the sum of the probabilities associated with these points. Note that as long as the sequences have long common tail, the corresponding values of $K_{2}$ are getting closer and closer. For simplicity we only track one path for the time being. Then we have

$$
\begin{aligned}
& a_{i+1}=p_{E}\left(z_{i+1}\right)\left(\pi_{00} a_{i}+\pi_{10} b_{i}\right) \\
& b_{i+1}=p_{E}\left(\bar{z}_{i+1}\right)\left(\pi_{01} a_{i}+\pi_{11} b_{i}\right) .
\end{aligned}
$$

It follows that

$$
\left(a_{i+1}+b_{i+1}\right) \leq \rho\left(a_{i}+b_{i}\right)
$$

here $0<\rho<1$ and $\rho$ is defined as

$$
\begin{aligned}
\rho=\max \left\{(1-\varepsilon) \pi_{00}+\varepsilon \pi_{01},(1-\varepsilon) \pi_{10}+\varepsilon \pi_{11}, \varepsilon \pi_{00}\right. \\
\left.+(1-\varepsilon) \pi_{01}, \varepsilon \pi_{10}+(1-\varepsilon) \pi_{11}\right\} .
\end{aligned}
$$

Immediately, we have

$$
\left(a_{n}+b_{n}\right) \leq \rho^{n} .
$$

Taking the derivative, we have

$$
\begin{aligned}
& a_{n+1}^{\prime}=-\left(\pi_{00} a_{n}+\pi_{10} b_{n}\right)+(1-\varepsilon)\left(\pi_{00} a_{n}^{\prime}+\pi_{10} b_{n}^{\prime}\right) \\
& b_{n+1}^{\prime}=\left(\pi_{01} a_{n}+\pi_{11} b_{n}\right)+\varepsilon\left(\pi_{10} a_{n}^{\prime}+\pi_{11} b_{n}^{\prime}\right) .
\end{aligned}
$$

In this case, we obtain

$$
\left|a_{n+1}^{\prime}\right|+\left|b_{n+1}^{\prime}\right| \leq \rho\left(\left|a_{n}^{\prime}\right|+\left|b_{n}^{\prime}\right|\right)+\rho^{n}
$$

which implies that there is a positive constant $C$ and $\rho_{1}$ with $\rho<\rho_{1}<$ 1 such that

$$
a_{n}^{\prime}+b_{n}^{\prime} \leq C \rho_{1}^{n} \text {. }
$$

Then we conclude that $\left|a_{n}^{\prime}+b_{n}^{\prime}\right| \rightarrow 0$ as $n \rightarrow \infty$. Exactly the same derivation can be applied to multiple path; it follows that

$$
q_{n} \leq \rho^{n}, \quad q_{n}^{\prime} \leq C \rho_{1}^{n} .
$$

So no matter which level we started from the deleted intervals, as long as they have long common tails, the corresponding values of $K_{2}$ function are close. Therefore, $K_{2}$ is well defined. 


\section{APPENDIX E \\ COMPUTATION OF $\left.H^{\prime \prime}(Z)\right|_{\varepsilon=1 / 2}$}

In this appendix, we basically follow the framework of [6]. Let

$$
\boldsymbol{p}_{n}=\left[p\left(Z_{1}^{n}, E_{n}=0\right), p\left(Z_{1}^{n}, E_{n}=1\right)\right]
$$

and

$$
\boldsymbol{M}\left(Z_{n-1}, Z_{n}\right)=\left[\begin{array}{cc}
(1-\varepsilon) p_{X}\left(Z_{n} \mid Z_{n-1}\right) & \varepsilon p_{X}\left(\bar{Z}_{n} \mid Z_{n-1}\right) \\
(1-\varepsilon) p_{X}\left(Z_{n} \mid \bar{Z}_{n-1}\right) & \varepsilon p_{X}\left(\bar{Z}_{n} \mid \bar{Z}_{n-1}\right)
\end{array}\right] .
$$

Then we have

$$
\boldsymbol{p}_{n}=\boldsymbol{p}_{n-1} \boldsymbol{M}\left(Z_{n-1}, Z_{n}\right) .
$$

Immediately, we obtain

$$
p_{Z}\left(Z_{1}^{n}\right)=\boldsymbol{p}_{1} \boldsymbol{M}\left(Z_{1}, Z_{2}\right) \cdots \boldsymbol{M}\left(Z_{n-1}, Z_{n}\right) \mathbf{1} .
$$

We consider the case when the channel is operating on the low-SNR region. For convenience, we let

$$
\delta=\frac{1}{2}-\varepsilon .
$$

Thus, when the SNR is very low, namely, $\varepsilon \rightarrow \frac{1}{2}$, correspondingly we have $\delta \rightarrow 0$. Since $H(Z)$ is an even function at $\delta=0$, the odd-order derivatives at $\delta=0$ are all equal to 0 . In the sequel, we shall compute the second derivative of $H(Z)$ at $\delta=0$.

In this case, we can rewrite the random matrix $\boldsymbol{M}_{i}=\boldsymbol{M}\left(z_{i} z_{i+1}\right)$ in the following way:

$$
\begin{aligned}
\boldsymbol{M}_{i}=\frac{1}{2}\left[\begin{array}{ll}
p_{X}\left(z_{i+1} \mid z_{i}\right) & p_{X}\left(\bar{z}_{i+1} \mid z_{i}\right) \\
p_{X}\left(z_{i+1} \mid \bar{z}_{i}\right) & p_{X}\left(\bar{z}_{i+1} \mid \bar{z}_{i}\right)
\end{array}\right] \\
+\delta\left[\begin{array}{ll}
p_{X}\left(z_{i+1} \mid z_{i}\right) & -p_{X}\left(\bar{z}_{i+1} \mid z_{i}\right) \\
p_{X}\left(z_{i+1} \mid \bar{z}_{i}\right) & -p_{X}\left(\bar{z}_{i+1} \mid \bar{z}_{i}\right)
\end{array}\right] .
\end{aligned}
$$

For the special case when $i=0$, we have

$$
M_{0}=\frac{1}{2}\left[p_{X}\left(z_{1}\right), p_{X}\left(\bar{z}_{i+1}\right)\right]+\delta\left[p_{X}\left(z_{1}\right),-p_{X}\left(\bar{z}_{1}\right)\right] .
$$

Then

$$
\begin{aligned}
& p_{Z}\left(z_{1}^{n}\right) \\
& \quad=\left(\frac{1}{2} \boldsymbol{M}_{0}^{(0)}+\delta \boldsymbol{M}_{0}^{(1)}\right)\left(\frac{1}{2} \boldsymbol{M}_{1}^{(0)}+\delta \boldsymbol{M}_{1}^{(1)}\right) \cdots\left(\frac{1}{2} \boldsymbol{M}_{n-1}^{(0)}+\delta \boldsymbol{M}_{n-1}^{(1)}\right) \mathbf{1} .
\end{aligned}
$$

Now define the function

$$
\mathbf{R}_{\mathbf{n}}(\delta)=\sum_{z_{1}^{n}} p_{Z}\left(z_{1}^{n}\right) \log \left(p_{Z}\left(z_{1}^{n}\right)\right) .
$$

Then according to the definition of $H(Z)$

$$
H(Z)=-\lim _{n \rightarrow \infty} \frac{1}{n} \boldsymbol{R}_{n}(\delta) .
$$

It can be checked that

$$
\left.\frac{\partial \boldsymbol{R}_{n}(\delta)}{\partial \delta}=\sum_{z_{1}^{n}} \frac{\partial p_{Z}\left(z_{1}^{n}\right)}{\partial \delta}\left(\log p_{Z}\left(z_{1}^{n}\right)+1\right)\right) .
$$

Now

$$
\begin{aligned}
& \left.\frac{\partial p_{Z}\left(z_{1}^{n}\right)}{\partial \delta}\right|_{\delta=0} \\
& =\left(\frac{1}{2}\right)^{n-1} \sum_{i=0}^{n-1} \boldsymbol{M}_{0}^{(0)} \boldsymbol{M}_{1}^{(0)} \cdots \boldsymbol{M}_{i-1}^{(0)} \boldsymbol{M}_{i}^{(1)} \boldsymbol{M}_{i+1}^{(0)} \cdots \boldsymbol{M}_{n-1}^{(0)} \mathbf{1} \\
& =\left(\frac{1}{2}\right)^{n-1} \sum_{i=1}^{n}\left(p_{X}\left(z_{i}\right)-p_{X}\left(\bar{z}_{i}\right)\right) .
\end{aligned}
$$

Again, simple calculations will lead to the first equation at the bottom of the page. Since $\left.\frac{\partial^{2} p_{Z}\left(z_{1}^{n}\right)}{\partial \delta^{2}}\right|_{\delta=0}$ is as defined in the second equation at the bottom of the page, we have

$$
\left.\frac{\partial^{2} \boldsymbol{R}_{n}(\delta)}{\partial \delta^{2}}\right|_{\delta=0}=\sum_{z_{1}^{n}} 2^{n}\left(\left(\frac{1}{2}\right)^{n-1} \sum_{i=1}^{n}\left(p_{X}\left(z_{i}\right)-p_{X}\left(\bar{z}_{i}\right)\right)\right)^{2} .
$$

Let $x, y$ temporarily denote the stationary distribution

$$
p_{X}(0)=\frac{\pi_{10}}{\pi_{01}+\pi_{10}}, \quad p_{X}(1)=\frac{\pi_{01}}{\pi_{01}+\pi_{10}}
$$

respectively. Then we get the equation at the top of the following page. Using the following two combinatoric identities:

$$
\sum_{i=0}^{n} i C_{n}^{i}=n 2^{n-1}
$$

and

$$
\sum_{i=0}^{n} i^{2} C_{n}^{i}=n(n-1) 2^{n-2}+n 2^{n-1},
$$

we derive

$$
\begin{aligned}
\left.\frac{\partial^{2} \boldsymbol{R}_{n}(\delta)}{\partial \delta^{2}}\right|_{\delta=0}= & \frac{1}{2^{n-2}}\left((x-y)^{2}\left(n(n-1) 2^{n}+n 2^{n+1}\right)\right. \\
& \left.+n^{2} 2^{n}(2 y-1)^{2}+2(x-y)(2 y-1) n^{2} 2^{n}\right) \\
= & 4 n(x-y)^{2}
\end{aligned}
$$

$$
\frac{\partial^{2} \boldsymbol{R}_{n}(\delta)}{\partial \delta^{2}}=\sum_{z_{1}^{n}}\left(\frac{\partial^{2} p_{Z}\left(z_{1}^{n}\right)}{\partial \delta^{2}} \log p_{Z}\left(z_{1}^{n}\right)+\frac{1}{p_{Z}\left(z_{1}^{n}\right)}\left(\frac{\partial p_{Z}\left(z_{1}^{n}\right)}{\partial \delta}\right)^{2}+\frac{\partial^{2} p_{Z}\left(z_{1}^{n}\right)}{\partial \delta^{2}}\right)
$$

$$
\begin{aligned}
\left.\frac{\partial^{2} p_{Z}\left(z_{1}^{n}\right)}{\partial \delta^{2}}\right|_{\delta=0} & =\left(\frac{1}{2}\right)^{n-2} \sum_{i \neq j} \boldsymbol{M}_{0}^{(0)} \boldsymbol{M}_{1}^{(0)} \cdots \boldsymbol{M}_{i-1}^{(0)} \boldsymbol{M}_{i}^{(1)} \boldsymbol{M}_{i+1}^{(0)} \cdots \boldsymbol{M}_{j-1}^{(0)} \boldsymbol{M}_{j}^{(1)} \boldsymbol{M}_{j+1}^{(0)} \cdots \boldsymbol{M}_{n-1}^{(0)} \mathbf{1} \\
& =\left(\frac{1}{2}\right)^{n-2}\left[p_{X}\left(z_{i+1}\right),-p_{X}\left(\bar{z}_{i+1}\right)\right]\left[\begin{array}{ll}
p_{X}\left(z_{j+1} \mid z_{i+1}\right) & -p_{X}\left(\bar{z}_{j+1} \mid z_{i+1}\right) \\
p_{X}\left(z_{j+1} \mid \bar{z}_{i+1}\right) & -p_{X}\left(\bar{z}_{j+1} \mid \bar{z}_{i+1}\right)
\end{array}\right] \\
& =\left(\frac{1}{2}\right)^{n-2} \sum_{i \neq j}\left(p_{X}\left(z_{j+1}, z_{i+1}\right)-p_{X}\left(z_{j+1}, \bar{z}_{i+1}\right)-p_{X}\left(\bar{z}_{j+1}, z_{i+1}\right)+p_{X}\left(\bar{z}_{j+1}, \bar{z}_{i+1}\right)\right)
\end{aligned}
$$




$$
\begin{aligned}
\left.\frac{\partial^{2} \boldsymbol{R}_{n}(\delta)}{\partial \delta^{2}}\right|_{\delta=0} & =\frac{1}{2^{n-2}} \sum_{i=0}^{n} C_{n}^{i}(2 i x+2(n-i) y-n)^{2} \\
& =\frac{1}{2^{n-2}} \sum_{i=0}^{n} C_{n}^{i}((2 x-2 y) i+2 n y-n)^{2} \\
& =(2 x-2 y)^{2} \sum_{i=0}^{n} C_{n}^{i} i^{2}+(2 n y-n)^{2} \sum_{i=0}^{n} 1+2(2 x-2 y)(2 n y-n) \sum_{i=0}^{n} C_{n}^{i} i
\end{aligned}
$$

From the fact that the derivatives of $H(Z)$ with respect to $\varepsilon$ are uniformly bounded on $[0,1 / 2]$ (see [6], also implied by Theorem 1.1 of [4] and the computation of $\left.H^{\varepsilon}(Z)\right|_{\varepsilon=0}$ ), we draw the conclusion that the second coefficient of $H(Z)$ is equal to

$$
\left.H^{\prime \prime}(Z)\right|_{\varepsilon=1 / 2}=-4\left(\frac{\pi_{10}-\pi_{01}}{\pi_{10}+\pi_{01}}\right)^{2} .
$$

\section{ACKNOWLEDGMENT}

The authors wish to thank the anonymous reviewer for pointing out the Faa Di Bruno formula, which greatly simplified the proof of Lemma 2.3.

\section{REFERENCES}

[1] D. Blackwell, "The entropy of functions of finite-state Markov chains," in Trans. 1st Prague Conf. Information Thoery, Statistical Decision Functions, Random Processes, Prague, Czechoslovakia, 1957, pp. $13-20$.

[2] G. Constantine and T. Savits, "A multivariate Faa Di Bruno formula with applications," Trans. Amer. Math. Soc., vol. 348, no. 2, pp. 503-520, Feb. 1996.

[3] R. Gharavi and V. Anantharam, "An upper bound for the largest Lyapunov exponent of a Markovian product of nonnegative matrices," Theor. Comp. Sci., vol. 332, no. 1-3, pp. 543-557, Feb. 2005.

[4] G. Han and B. Marcus, "Analyticity of entropy rate of hidden Markov chains," IEEE Trans. Inf. Theory, vol. 52, no. 12, pp. 5251-5266, Dec. 2006.

[5] T. Holliday, A. Goldsmith, and P. Glynn, "Capacity of finite state channels based on Lyapunov exponents of random matrices," IEEE Trans. Inf. Theory, vol. 52, no. 8, pp. 3509-3532, Aug. 2006.

[6] P. Jacquet, G. Seroussi, and W. Szpankowski, "On the entropy of a hidden Markov process," in Proc. Data Compression Conf., Snowbird, UT, Mar. 2004, pp. 362-371.

[7] J. Kemeny and J. Snell, Finite Markov Chains. Princeton, N.J.: Van Nostrand, 1960.

[8] R. Leipnik and T. Reid, "Multivariable Faa Di Bruno formulas," in Electronic Proc 9th Annu. Int. Conf. Technology in Collegiate Mathematics [Online]. Available: http://archives.math.utk.edu/ICTCM/EP-9. html\#C23

[9] D. Lind and B. Marcus, An Introduction to Symbolic Dynamics and Coding. Cambridge, U.K.: Cambridge Univ. Press, 1995.

[10] B. Marcus, K. Petersen, and S. Williams, "Transmission rates and factors of Markov chains," Contemp. Math., vol. 26, pp. 279-294, 1984.

[11] E. Ordentlich and T. Weissman, "On the optimality of symbol by symbol filtering and denoising," IEEE Trans. Inf. Theory, vol. 52, no. 1, pp. 19-40, Jan. 2006.

[12] E. Ordentlich and T. Weissman, "New bounds on the entropy rate of hidden Markov process," in Proc. Information Theory Workshop, San Antonio, TX, Oct. 2004, pp. 117-122.

[13] E. Ordentlich and T. Weissman, Personal Communication.

[14] Y. Peres, "Analytic dependence of Lyapunov exponents on transition probabilities," in Lyapunov's Exponents, Proceedings of a Workshop (Lecture Notes in Mathematics). Berlin, Germany: Springer-Verlag, 1990, vol. 1486.

[15] Y. Peres, "Domains of analytic continuation for the top Lyapunov exponent," Ann. Inst. H. Poincaré Probab. Statist., vol. 28, no. 1, pp. 131-148, 1992.
[16] O. Zuk, I. Kanter, and E. Domany, "Asymptotics of the entropy rate for a hidden Markov process,” J. Statist. Phys., vol. 121, no. 3-4, pp. 343-360, 2005.

[17] O. Zuk, E. Domany, I. Kanter, and M. Aizenman, "From finite-system entropy to entropy rate for a hidden Markov process," IEEE Signal Process. Lett., , vol. 13, no. 9, pp. 517-520, Sep. 2006.

\section{The Fading Number of Memoryless Multiple-Input Multiple-Output Fading Channels}

Stefan M. Moser, Member, IEEE

\begin{abstract}
In this correspondence, we derive the fading number of multiple-input multiple-output (MIMO) flat-fading channels of general (not necessarily Gaussian) regular law without temporal memory. The channel is assumed to be noncoherent, i.e., neither receiver nor transmitter have knowledge about the channel state, but they only know the probability law of the fading process. The fading number is the second term, after the double-logarithmic term, of the high signal-to-noise ratio (SNR) expansion of channel capacity. Hence, the asymptotic channel capacity of memoryless MIMO fading channels is derived exactly. The result is then specialized to the known cases of single-input-multiple-output (SIMO), multiple-input single-output (MISO), and single-input-single-output (SISO) fading channels, as well as to the situation of Gaussian fading.
\end{abstract}

Index Terms-Channel capacity, fading number, Gaussian fading, general flat fading, high signal-to-noise ratio (SNR), multiple antenna, multiple-input multiple-output (MIMO), noncoherent.

\section{INTRODUCTION}

It has been recently shown in [1], [2] that, whenever the matrixvalued fading process is of finite differential entropy rate (a so-called regular process), the capacity of noncoherent multiple-input multipleoutput (MIMO) fading channels typically grows only double-logarithmically in the signal-to-noise ratio (SNR).

This is in stark contrast to both, the coherent fading channel where the receiver has perfect knowledge about the channel state, and to the noncoherent fading channel with nonregular channel law, i.e., the differential entropy rate of the fading process is not finite. In the former case the capacity grows logarithmically in the SNR with a

Manuscript received June 1, 2006; revised March 12, 2007. This work was supported by the Industrial Technology Research Institute (ITRI), Zhudong, Taiwan, under Contract G1-95003.

The author is with the Department of Communication Engineering, National Chiao Tung University (NCTU), Hsinchu, Taiwan (e-mail: stefan.moser@ieee. org).

Communicated by K. Kobayashi, Associate Editor for Shannon Theory.

Digital Object Identifier 10.1109/TIT.2007.899512 